\title{
Environment-Centric Contracts for Design of Cyber-Physical Systems
}

\author{
Jonas Westman ${ }^{1}$ and Mattias Nyberg ${ }^{2}$ \\ 1 Royal Institute of Technology (KTH), Stockholm, Sweden \\ jowestm@kth.se \\ 2 Scania, Södertälje, Sweden
}

\begin{abstract}
A contract splits the responsibilities between a component and its environment into a guarantee that expresses an intended property under the responsibility of the component, given that the environment fulfills the assumptions. Although current contract theories are limited to express contracts over interfaces of components, specifications that are not limited to interfaces are used in practice and are needed in order to properly express safety requirements. A framework is therefore presented, generalizing current contract theory to environment-centric contracts - contracts that are not limited to the interface of components. The framework includes revised definitions of properties of contracts, as well as theorems that specifies exact conditions for when the properties hold. Furthermore, constraints are introduced, limiting the ports over which an environment-centric contract is expressed where the constraints constitute necessary conditions for the guarantee of the contract to hold in an architecture.
\end{abstract}

Keywords: Environment-Centric, Contracts, Architecture

\section{Introduction}

The notion of contracts was first introduced in [1] as a pair of pre- and postconditions $[2-5]$ to formally specify the interface of software components. However, in more recent work [6-9] where the use of contracts is extended to the design of Cyber-Physical Systems (CPS) [10], the conceptual idea of a contract is rather described as: "a component model that sets forth the assumptions under which the component may be used by its environment, and the corresponding promises that are guaranteed under such correct use" [6], which indicates that contracts must not necessarily be limited to the interfaces of components.

However, in current contract theories [1,5-9,11-21], contracts are indeed limited to the interface of components, e.g. as shown in Fig. 1a where a contract for a controller $\mathbb{C}_{1}$ is limited to its interface. In this case, the guarantee $G$ expresses that the desired output signal $v$ to another controller $\mathbb{C}_{2}$ is a function of the voltage $u$ at an input pin connected to a sensor $\mathbb{S}$. However, the guarantee G can only be assured to hold, given that the assumption A is fulfilled where A expresses constraints on the input $u$. In contrast to Fig. 1a, Fig. 1b shows 


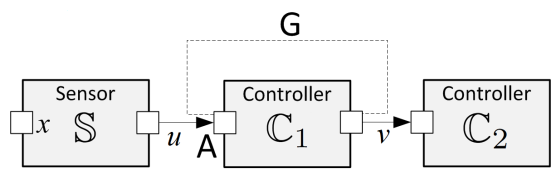

(a)

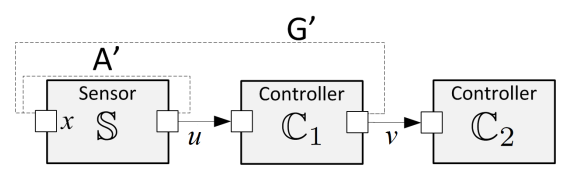

(b)

Fig. 1. Two contracts for a controller $\mathbb{C}_{1}$ are shown in (a) and (b), where the contract in (a) is limited to the interface of $\mathbb{C}_{1}$ and the contract in (b), is not.

another contract for $\mathbb{C}_{1}$ where both the guarantee $G^{\prime}$ and the assumption $A^{\prime}$ are not limited to the interface of $\mathbb{C}_{1}$. In this case, the guarantee $G^{\prime}$ expresses that the output signal $v$ shall correspond to a physical quantity $x$, as sensed by the sensor $\mathbb{S}$, given the assumption $A$ that sensor $\mathbb{S}$ is functioning correctly.

In order to support specifications that are not limited to the interfaces of components, e.g. the one shown in Fig. 1b, the present paper generalizes current contract theories $[1,5-9,11-21]$ to contracts that are not limited to the interface of components. Inspired by $[22,23]$, a contract that is not limited to the interface of a component, will in the following be referred to as an environment-centric contract.

It could be argued that the environment-centric contract in Fig. 1b is not needed since the interface of $\mathbb{C}_{1}$ could be extended to include the port $x$, which would allow the contract in Fig. $1 b$ to be a contract for $\mathbb{C}_{1}$. However, in this case, the interface of the component $\mathbb{C}_{1}$ would not match the interface of the real world object that it models and would hence require the use of ambiguous representations of the real world. The approach can hence be discharged and it can be concluded that environment-centric contracts are not supported by current contract theories $[1,5-9,11-21]$.

Although environment-centric contracts are not supported by current contract theories, there are at least two reasons why a generalization of current contract theories to environment-centric contracts is strongly needed.

The first reason is that a specification, which is not limited to the interface of a component, is capable of expressing that the responsibility of the component is to achieve an overall intended property of a system, instead of being restricted to express only its intended behavior $[22,23]$. For example, the environmentcentric contract in Fig. 1b, enables to express that it is the responsibility of $\mathbb{C}_{1}$ to guarantee that the output $v$ is an accurate estimation of the physical quantity $x$, sensed by the sensor, instead of being limited to guarantee that the output signal $v$ is a function of the voltage $u$ at the pin as shown in Fig. 1a. An example of when a specification such as the one shown in Fig. 1b is used in an industrial case-study, can be found in [24] where ModelicaML [25] is used to specify and verify requirements on a subsystem of a fuel management system where the requirements express the end-to-end functionality of the fuel management system in general. Another example can be found in [26] where SysML [27] is used to specify requirements on an engine knock controller and where the requirements 
allocated to the controller explicitly refer to parts, such as e.g. the piston, of the environment of the controller.

The second reason why environment-centric contracts are needed is that, in the area of functional safety $[28,29]$, the associated risk of a component, is assessed in the context of how it affects its environment, and not just by its immediate behavior. In order to properly express safety requirements on a component, there is hence a need to refer to parts in the environment that the component is to be deployed in. For example, in ISO 26262 [29], top-level safety requirements for an item, i.e. a system, are formulated in order to prevent or mitigate hazards, where the hazards "shall be defined in terms of the conditions or behaviour that can be observed at the vehicle level" [29]. This can be observed in the industrial examples $[30,31]$, where requirements that are not limited to the interface of components are necessarily used in order to properly express safety requirements on the components.

The two reasons above explain the importance of allowing assumptions and guarantees to be expressed, not only over the interface, but also over ports in the environment. This motivates the main contribution of this paper, namely a framework that generalizes current contract theories to environment-centric contracts.

At the core of the framework is a corollary that, given an environment-centric contract, cleanly separates the respective conditions that a component and its environment need to meet in order to ensure seamless integration into a final product where the guarantee of the environment-centric contract holds. Considering such conditions, necessary constraints on the set of ports, i.e. the scopes, over which guarantees and assumptions can be expressed, are introduced. The constraints serve as a sanity check in order to determine that an environmentcentric contract is not an unreasonable specification for a component in an architecture.

The framework includes revised definitions of the contract properties consistency $[6,7,20]$, compatibility $[6,7,20]$, and dominance $[8,21]$ as defined in current contract theories, as well as two theorems that specify necessary and sufficient conditions of consistency and compatibility. As a basis for structuring contracts in parallel to an architecture, a graph, called a decomposition structure, is introduced. Based on a decomposition structure, a theorem is presented with sufficient conditions of dominance.

Out of an extensive literature study of contract theories $[1,5-9,11-17,19-21]$, no previous contract theories were found to explicitly support contracts that are not limited to the interface of components. Although both [20,21] do allow assumptions that extend outside of the interface, both are, however, limited to express guarantees over the interface of components. Even though the abstract definition of a contract in [8] does not exclude that assumptions and the guarantees are limited to the interface of components, it is not mentioned explicitly. Moreover, [8] does not address compatibility and consistency issues between interfaces. In contrast to $[8,20,21]$, the present paper focuses on fully generalizing 
contract theories by revising properties of contracts and providing theorems to facilitate practical application.

Although the work in the present paper is a generalization of current contract theories $[1,5-9,11-21]$ in general, due to the numerous contract theories that exist, the text is limited to discuss properties of contracts as presented in contract theories [6-8,21] that can be traced back to the FP6 project SPEEDS [32]. This means that, e.g. the quotient operator $[9,20]$, is not discussed. The work is further confined to representing assumptions and guarantees as sets of runs, which means that neither modalities $[8,19]$, nor contracts that support probabilities $[17,18]$, are included.

\section{Assertions, Elements and Architectures}

This section establishes a theoretic framework in order to model a CPS and its parts, and to be able to describe the notion of environment-centric contracts and its properties in Sec. 3 and 4. The framework mainly draws inspiration from the contract theory of the FP6 project SPEEDS [32] as described in $[6,7]$.

\subsection{Assertions and Runs}

Let $X=\left\{x_{1}, \ldots, x_{N}\right\}$ be a set of variables. Consider a trajectory of values of a variable $x_{i}$ over a time window starting at a certain time $t_{0}$. A tuple of such trajectories, one for each variable in $X$, sorted according to a global ordering with respect to the identifiers of the variables, is called a run for $X$, denoted $\omega_{X}$. Given a set of variables $X^{\prime}$, an assertion $\mathrm{W}$ over $X^{\prime}$ is a possibly empty set of runs for $X^{\prime}$. This notion corresponds to similar definitions in $[6,7,31]$. Note that, in the following, assertions will be specified by equations.

Given an assertion $\mathrm{W}$ over $X=\left\{x_{1}, \ldots, x_{N}\right\}$, and another set of variables $X^{\prime} \subseteq X$, the projection $[6,20,21,31]$ of $\mathrm{W}$ onto $X^{\prime}$, written $\operatorname{proj}_{X^{\prime}}(\mathrm{W})$, is the set of runs obtained when the trajectory of values of each variable $x_{i} \notin X^{\prime}$ is removed from each run in W. Using notation of relational algebra [33], it holds that

$$
\operatorname{proj}_{X^{\prime}}(\mathrm{W})=\pi_{X^{\prime}}(\mathrm{W}) .
$$

Given an assertion $\mathrm{W}^{\prime}$ over $X^{\prime}$ and another set of variables $X, \widehat{\operatorname{proj}}_{X}\left(\mathrm{~W}^{\prime}\right)$ is the set of runs where each run in $W^{\prime}$ is first extended with all possible runs for $X \backslash X^{\prime}$, prior to applying the operation of projection. That is,

$$
\widehat{\operatorname{proj}}_{X}\left(\mathrm{~W}^{\prime}\right)=\operatorname{proj}_{X}\left(\left\{\omega_{X \cup X^{\prime}} \mid \operatorname{proj}_{X^{\prime}}\left(\left\{\omega_{X \cup X^{\prime}}\right\}\right) \in \mathrm{W}^{\prime}\right\}\right) .
$$

In the following, the symbols $\widehat{\cap}, \widehat{\cup}, \widehat{C}, \widehat{\subseteq}$, etc. will be used to denote that prior to using operations and relations on assertions over dissimilar sets of variables, the assertions are first extended to the union of the sets of variables involved using the operator $\widehat{\operatorname{proj}}$. For example, given two assertions $\mathrm{W}$ and $\mathrm{W}^{\prime}$ over the set of variables $X$ and $X^{\prime}$, respectively, $\mathrm{W} \widehat{\cap} \mathrm{W}^{\prime}=\widehat{\operatorname{proj}}_{X \cup X^{\prime}}(\mathrm{W}) \cap \widehat{\operatorname{proj}}_{X \cup X^{\prime}}\left(\mathrm{W}^{\prime}\right)$. 
Let $\Omega_{X}$ denote the set of all possible runs for a set of variables $X$. An assertion W over $X$ constrains a set of variables $X^{\prime}$ if for each $x^{\prime} \in X^{\prime}$, it holds that

$$
\begin{cases}\mathrm{W} \widehat{\subset} \operatorname{proj}_{X \backslash\left\{x^{\prime}\right\}}(\mathrm{W}) & X \backslash\left\{x^{\prime}\right\} \neq \emptyset \\ \mathrm{W} \subset \Omega_{\left\{x^{\prime}\right\}} & \text { otherwise. }\end{cases}
$$

\subsection{Elements}

In this section, the concept of elements, corresponding to Heterogeneous Rich Component (HRC) in [6,7], is introduced in order to model any entity of a CPS in general, such as software, hardware, or physical entities, as well as to serve as a functional or logical design entity in general, e.g. as a SysMl block [27].

Definition 1 (Element). An element $\mathbb{E}$ is an ordered pair $(X, \mathrm{~B})$ where:

a) $X$ is a set of variables, called the interface of $\mathbb{E}$ and where each $x \in X$ is called a port variable; and

b) $\mathrm{B}$ is an assertion over $X$, called the behavior of $\mathbb{E}$.

Port variables model tangible quantities of the element from the perspective of an external observer to the entity, and the behavior models the static and dynamic constraints that the element imposes on the port variables, independent of its surroundings.

As an illustrative example, consider a potentiometer $\mathbb{E}_{\text {pot }}=\left(X_{\text {pot }}, \mathrm{B}_{\text {pot }}\right)$ where $X_{\text {pot }}=\left\{v_{\text {ref }}, v_{\text {branch }}, v_{\text {gnd }}\right\}$. The port variables $v_{\text {ref }}, v_{\text {branch }}$, and $v_{\text {gnd }}$ model the reference, branch, and ground voltages, respectively. Furthermore, $h$ models the position $(0-100 \%)$ of the 'slider' that moves over the resistor and branches the circuit. Given a simplified model where currents are neglected, the behavior $\mathrm{B}_{\text {pot }}$ can be specified by the equation $h=\frac{v_{\text {branch }}-v_{g n d}}{v_{\text {ref }}-v_{\text {gnd }}}$.

\subsection{Architectures}

This section describes how a set of elements can be structured in order to model a CPS, its parts, and its surroundings. Similar to e.g. [29, 34], such a structure will be referred to as an architecture, which, in this paper, will be denoted with the symbol $\mathscr{A}$. In contrast to other contract theories, e.g. [6-8], the present paper focuses more on the structuring of elements and on the sharing of port variables. A well-defined structure of how ports are shared is needed in order to define scoping constraints for environment-centric contracts in Sec. 3.2.

Prior to presenting the formal definition of an architecture, the concept is introduced informally by describing an architecture $\mathscr{A}_{L M-s y s}$ of a "Level Meter system" (LM-system) $\mathbb{E}_{L M-s y s}$, as shown in Fig. 2a where an element is represented as a rectangle filled with gray with boxes on its edges that symbolize its port variables and in Fig. $2 \mathrm{~b}$ where the hierarchical structure of the LM-system is shown as a tree. 
As shown in Fig. 2a, the LM-system $\mathbb{E}_{L M-s y s}$ consists of a tank $\mathbb{E}_{\text {tank }}$ and an electric-system $\mathbb{E}_{E-\text { sys }}$. The electric-system $\mathbb{E}_{E \text {-sys }}$ consists of the potentiometer $\mathbb{E}_{\text {pot }}$ as described in Sec. 2.2, a battery $\mathbb{E}_{\text {bat }}$ and a level meter $\mathbb{E}_{l \text { Meter }}$. The slider $h$ is connected to a "floater", trailing the level $f$ in the tank. In this way, the potentiometer $\mathbb{E}_{\text {pot }}$ is used as a level sensor to estimate the level in the tank. The estimated level is presented by the level meter $\mathbb{E}_{l \text { Meter }}$ where $l$ denotes the presented level.

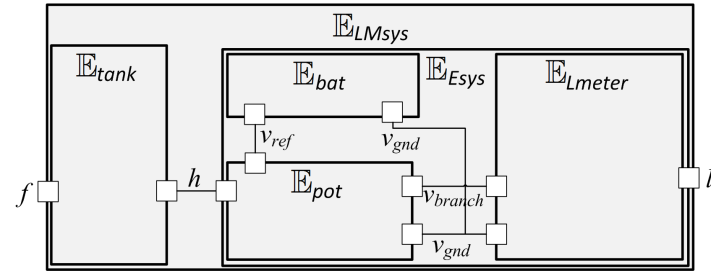

(a)

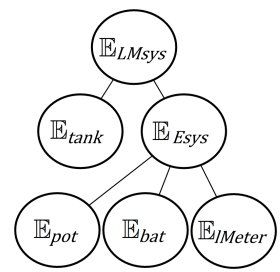

(b)

Fig. 2. An architecture $\mathscr{A}_{L M-\text { sys }}$ of a "Level Meter system".

Notably, when examining each part of the LM-system individually, each part will have quantities that may not be perceivable when the parts are integrated with each other. That is, the port variable $v_{r e f}$, for example, is not part of the interface of $\mathbb{E}_{E s y s}$ and is hence considered to be a quantity of the E-system that is not perceivable to an external observer to the E-system. To capture such a notion it is enforced that any port variable $x$ of a child of an element $\mathbb{E}=(X, \mathrm{~B})$ where $x \notin X$ cannot be a member of an interface of a non-descendant of $\mathbb{E}$.

Consider that the behavior $\mathrm{B}_{b a t}$ of $\mathbb{E}_{b a t}$ is the set of all runs where $v_{r e f}-$ $v_{\text {gnd }}=5 \mathrm{~V}$ and that the behavior $\mathrm{B}_{\text {Lmeter }}$ of $\mathbb{E}_{\text {Lmeter }}$ is the set of all runs where $l=\frac{v_{\text {branch }}-v_{\text {gnd }}}{5}$. In order to relate the individual behaviors of the children of $\mathbb{E}_{\text {Esys }}$ with the behavior of $\mathbb{E}_{\text {Esys }}$, the individual behaviors need to be combined with each other and subsequently restricted to the interface of $\mathbb{E}_{E s y s}$. This can be achieved by relying on the intersection operator and the projection operator, that is, in accordance with the Sec. 2.1, $\mathrm{B}_{\text {Esys }}=\widehat{\operatorname{proj}}_{X_{\text {Esys }}}\left(\mathrm{B}_{\text {pot }} \widehat{\cap} \mathrm{B}_{\text {bat }} \widehat{\cap} \mathrm{B}_{\text {Lmeter }}\right)$.

Now that the concept of an architecture has been introduced informally, the formal definition follows.

Definition 2 (Architecture). An architecture $\mathscr{A}$ is a set of elements organized into a rooted tree, such that:

(a) for any non-leaf node $\mathbb{E}=(X, \mathrm{~B})$, with children $\left\{\left(X_{i}, \mathrm{~B}_{i}\right)\right\}_{i=1}^{N}$, it holds that $\mathrm{B}=\widehat{\operatorname{proj}}_{X}\left(\widehat{\bigcap}_{i=1}^{N} \mathrm{~B}_{i}\right) ;$ and

(b) if there is a child $\mathbb{E}^{\prime}=\left(X^{\prime}, \mathrm{B}^{\prime}\right)$ and a non-descendent $\mathbb{E}^{\prime \prime}=\left(X^{\prime \prime}, \mathrm{B}^{\prime \prime}\right)$ of $\mathbb{E}=(X, \mathrm{~B})$, such that $x \in X^{\prime}$ and $x \in X^{\prime \prime}$, then it holds that $x \in X$.

For convenience, in the context of an architecture, the environment of an element $\mathbb{E}$ is considered to be the set of elements in the surroundings of $\mathbb{E}$. That 
is, as shown in Fig 2 , the elements $\mathbb{E}_{\text {bat }}, \mathbb{E}_{\text {tank }}$, and $\mathbb{E}_{l \text { Meter }}$ are elements in the environment of the potentiometer $\mathbb{E}_{\text {pot }}$.

Definition 3 (Environment of Element). Given an architecture $\mathscr{A}$, the environment of an element $\mathbb{E}=(X, \mathrm{~B})$ in $\mathscr{A}$, denoted Env $v_{\mathscr{A}}(\mathbb{E})$, is the set of elements $\left\{\mathbb{E}_{i}\right\}_{i=1}^{N}$ such that $\mathbb{E}_{i}=\left(X_{i}, \mathrm{~B}_{i}\right)$ is either a sibling or a sibling of a proper ancestor of $\mathbb{E}$. Let $\mathrm{B}_{E n v_{\mathscr{A}}(\mathbb{E})}=\widehat{\bigcap}_{i=1}^{N} \mathrm{~B}_{i}$, denote the behavior of $E n v_{\mathscr{A}}(\mathbb{E})$.

Given that an assertion is possibly the empty set (See Sec. 2.1), an architecture $\mathscr{A}$ is considered to be realizable if the behavior of the root element of $\mathscr{A}$ is non-empty.

Definition 4 (Realizable Architecture). An architecture $\mathscr{A}$ is realizable if the behavior of the root element of $\mathscr{A}$ is non-empty.

Proposition 1. Given an architecture $\mathscr{A}$ containing an element $\mathbb{E}=(X, \mathrm{~B})$, it holds that $\mathscr{A}$ is realizable, if and only if $\mathrm{B}_{E_{n n v_{\mathscr{A}}}(\mathbb{E})} \widehat{\cap} \mathrm{B} \neq \emptyset$.

Proof. For the if part, consider an architecture $\mathscr{A}$ containing an element $\mathbb{E}=$ $(X, \mathrm{~B})$ where

$$
\mathrm{B}_{E n v_{\mathscr{A}}(\mathbb{E})} \widehat{\cap} \mathrm{B} \neq \emptyset .
$$

If $\left\{\mathbb{E}_{i}\right\}_{i=1}^{M}$ is the set of siblings of $\mathbb{E}$ and $\left\{\mathbb{E}_{i}\right\}_{i=M+1}^{N}$ is the set of siblings of proper ancestors of $\mathbb{E}$ where $\mathbb{E}_{i}=\left(X_{i}, \mathrm{~B}_{i}\right)$, then in accordance with Definition 3 it holds that $\mathrm{B}_{E n v_{\mathscr{A}}(\mathbb{E})}=\left(\widehat{\bigcap}_{i=M+1}^{N} \mathrm{~B}_{i}\right) \widehat{\cap}\left(\widehat{\bigcap}_{i=1}^{M} \mathrm{~B}_{i}\right)$. This and the relation (4) imply that

$$
\left(\widehat{\bigcap}_{i=M+1}^{N} \mathrm{~B}_{i}\right) \widehat{\cap}\left(\widehat{\bigcap}_{i=1}^{M} \mathrm{~B}_{i}\right) \widehat{\cap} \mathrm{B} \neq \emptyset .
$$

Since $\left(\widehat{\bigcap}_{i=1}^{M} \mathrm{~B}_{i}\right) \widehat{\cap} \mathrm{B}$ is an assertion over $\left(\bigcup_{i=1}^{M} X_{i}\right) \cup X$, Lemma 1 implies that

$$
\operatorname{proj}_{\left(\bigcup_{i=1}^{M} X_{i}\right) \cup X}\left(\left(\widehat{\bigcap}_{i=1}^{M} \mathrm{~B}_{i}\right) \widehat{\cap} \mathrm{B}\right)=\left(\widehat{\bigcap}_{i=1}^{M} \mathrm{~B}_{i}\right) \widehat{\cap} \mathrm{B} .
$$

This, Lemma 5 , and since $X \subseteq\left(\bigcup_{i=1}^{M} X_{i}\right) \cup X$ imply that

$$
\left(\widehat{\bigcap}_{i=1}^{M} \mathrm{~B}_{i}\right) \widehat{\cap} \mathrm{B} \widehat{\subseteq} \operatorname{proj}_{X}\left(\left(\widehat{\bigcap}_{i=1}^{M} \mathrm{~B}_{i}\right) \widehat{\cap} \mathrm{B}\right) \text {. }
$$

This can also be written as

$$
\left(\widehat{\bigcap}_{i=1}^{M} \mathrm{~B}_{i}\right) \widehat{\cap} \mathrm{B} \subseteq \widehat{\operatorname{proj}}_{\left(\bigcup_{i=1}^{M} X_{i}\right) \cup X}\left(\operatorname{proj}_{X}\left(\left(\widehat{\bigcap}_{i=1}^{M} \mathrm{~B}_{i}\right) \widehat{\cap} \mathrm{B}\right)\right) .
$$

This and the relation (5) imply that

$$
\emptyset \subset\left(\widehat{\bigcap}_{i=M+1}^{N} \mathrm{~B}_{i}\right) \widehat{\cap}\left(\widehat{\bigcap}_{i=1}^{M} \mathrm{~B}_{i}\right) \widehat{\cap} \mathrm{B} \subseteq
$$




$$
\left(\widehat{\bigcap}_{i=M+1}^{N} \mathrm{~B}_{i}\right) \widehat{\cap} \widehat{\operatorname{proj}}_{\left(\cup_{i=1}^{M} X_{i}\right) \cup X}\left(\operatorname{proj}_{X}\left(\left(\widehat{\bigcap}_{i=1}^{M} \mathrm{~B}_{i}\right) \widehat{\cap} \mathrm{B}\right)\right),
$$

In accordance with Sec. 2.1, this can also be written as

$$
\emptyset \subset\left(\widehat{\bigcap}_{i=M+1}^{N} \mathrm{~B}_{i}\right) \hat{\cap}\left(\widehat{\bigcap}_{i=1}^{M} \mathrm{~B}_{i}\right) \widehat{\cap} \mathrm{B} \widehat{\subseteq}\left(\widehat{\bigcap}_{i=M+1}^{N} \mathrm{~B}_{i}\right) \widehat{\cap} \operatorname{proj}_{X}\left(\left(\widehat{\bigcap}_{i=1}^{M} \mathrm{~B}_{i}\right) \widehat{\cap} \mathrm{B}\right)
$$

If $\mathbb{E}^{\prime}=\left(X^{\prime}, \mathrm{B}^{\prime}\right)$ is the parent of $\mathbb{E}$, in accordance with Definition 2 and 3 , it hence holds that

$$
\emptyset \subset \mathrm{B}_{E n v_{\mathscr{A}}(\mathbb{E})} \hat{\cap} \mathrm{B} \widehat{\subseteq} \mathrm{B}_{E n v_{\mathscr{A}}\left(\mathbb{E}^{\prime}\right)} \hat{\cap} \mathrm{B}^{\prime} .
$$

Since the same steps that were applied to $\mathbb{E}$ can be applied to $\mathbb{E}^{\prime}$ and then to the parent of $\mathbb{E}^{\prime}$, and so on, until the root node $\mathbb{E}_{r}=\left(X_{r}, \mathrm{~B}_{r}\right)$ is reached, and since $\operatorname{Env}_{\mathscr{A}}\left(\mathbb{E}_{r}\right)=\emptyset$, it holds that

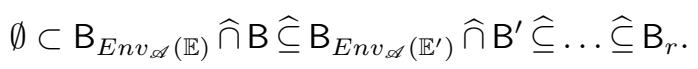

In accordance with Definition $4, \mathscr{A}$ is hence realizable, which completes the if part of the proof.

For the if-only part, assume that $\mathscr{A}$ is a realizable architecture. According to Definition 4 , this means that the behavior of the root node $\mathbb{E}_{r}=\left(X_{r}, \mathrm{~B}_{r}\right)$ is non-empty, i.e. that $\mathrm{B}_{r} \neq \emptyset$. If $\left\{\mathbb{E}_{i}\right\}_{i=1}^{N}$ is the complete set of children of $\mathbb{E}_{r}$ where $\mathbb{E}_{i}=\left(X_{i}, \mathrm{~B}_{i}\right)$, then, in accordance with Definition 2 , it hence holds that

$$
\widehat{\operatorname{proj}}_{X}\left(\widehat{\bigcap}_{i=1}^{N} \mathrm{~B}_{i}\right) \neq \emptyset \text {. }
$$

Consider an element $\mathbb{E}_{j} \in\left\{\mathbb{E}_{i}\right\}_{i=1}^{N}$. According to Definition 3, it holds that $\mathrm{B}_{E n v_{\mathscr{A}}\left(\mathbb{E}_{j}\right)}=\widehat{\bigcap}_{i=1, i \neq j}^{N} \mathrm{~B}_{i}$. This and the relation (6) imply that

$$
\widehat{\operatorname{proj}_{X}}\left(\mathrm{~B}_{E n v_{\mathscr{A}}\left(\mathbb{E}_{j}\right)} \widehat{\cap} \mathrm{B}_{j}\right) \neq \emptyset .
$$

This and Lemma 6 imply that

$$
\mathrm{B}_{E n v_{\mathscr{A}}\left(\mathbb{E}_{j}\right)} \hat{\cap} \mathrm{B}_{j} \neq \emptyset .
$$

In the following it will be shown that the relation (8) also holds for any proper descendant of $\mathbb{E}_{j}$.

If $\left\{\mathbb{E}_{j, i}\right\}_{i=1}^{M}$ is the complete set of children of $\mathbb{E}_{j}$ where $\mathbb{E}_{j, i}=\left(X_{j, i}, \mathrm{~B}_{j, i}\right)$, then, in accordance with Definition 2, it hence holds that

$$
\mathrm{B}_{j}=\widehat{\operatorname{proj}}_{X_{j}}\left(\widehat{\bigcap}_{i=1}^{M} \mathrm{~B}_{j, i}\right) \text {. }
$$

This, Lemma 6, and the relation (7) imply that

$$
\mathrm{B}_{E n v_{\mathscr{A}}\left(\mathbb{E}_{j}\right)} \widehat{\cap} \widehat{\operatorname{proj}}_{X_{j}}\left(\widehat{\bigcap}_{i=1}^{M} \mathrm{~B}_{j, i}\right) \neq \emptyset \text {. }
$$


This and Lemma 6 imply that

$$
\widehat{\bigcap}_{i=1}^{M} \mathrm{~B}_{j, i} \neq \emptyset
$$

In accordance with Definition 2 and 3, and Lemma 3, $\mathrm{B}_{E n v_{\mathscr{A}}\left(\mathbb{E}_{j}\right)}$ does not constrain $\left(\bigcup_{i=1}^{M} X_{j, i}\right) \backslash X_{j}$. In accordance with the relation (3), $\mathrm{B}_{E n v_{\mathscr{A}}\left(\mathbb{E}_{j}\right)}$ allows any variable in $\left(\bigcup_{i=1}^{M} X_{j, i}\right) \backslash X_{j}$ to have any value at any point in time. This and the relations (9) and (10) imply that

$$
\mathrm{B}_{E n v_{\mathscr{A}}\left(\mathbb{E}_{j}\right)} \widehat{\cap} \widehat{\bigcap}_{i=1}^{M} \mathrm{~B}_{j, i} \neq \emptyset .
$$

Consider a child $\mathbb{E}_{j, k}$ of $\mathbb{E}_{j}$. The relation (11) and since it holds, in accordance with Definition 3, that $\mathrm{B}_{E n v_{\mathscr{A}}\left(\mathbb{E}_{j, k}\right)}=\mathrm{B}_{E n v_{\mathscr{A}}\left(\mathbb{E}_{j}\right)} \widehat{\cap}\left(\widehat{\bigcap}_{i=1, i \neq k}^{M} \mathrm{~B}_{j, i}\right)$ imply that

$$
\mathrm{B}_{E n v_{\mathscr{A}}\left(\mathbb{E}_{j, k}\right)} \widehat{\cap} \mathrm{B}_{j, k} \neq \emptyset .
$$

Since the same steps that were applied to $\mathbb{E}_{j, k}$ can be applied on any child of $\mathbb{E}_{j, k}$, and so on, it holds that the relation (12) also holds for any descendant in $\mathscr{A}$. This and the relation (7) imply that the the intersection of the behavior of any element in $\mathscr{A}$ and the behavior of its environment is non-empty, which completes the if-only part of the proof.

\section{Environment-Centric Contracts}

As mentioned in Sec. 1, the notion of contracts was first introduced in [1] as a pair of pre and post-conditions, to be used as a specification in object-oriented programming. The principles behind contracts can, however, be traced back to early ideas on proof-methods [2-4] and compositional reasoning/verification [5, $11,12]$. Since then, several frameworks for compositional reasoning $[13,14]$ have emerged and also techniques to automate the approach have been proposed, see e.g. [35] or [36] for a survey. The work in [1] has been extended to e.g. component-based design [15] and analog systems [16].

In more recent work $[6-8,21]$, inspired by e.g. [37-40], the use of contracts is extended to serve as a central design philosophy in systems engineering to support the design of CPS. As mentioned in Sec. 1, in a context of CPS design, the conceptual idea of a contract does not prescribe that a contract must necessarily be limited to the interface of an element, thus allowing environment-centric contracts. Considering this, an environment-centric contract will, in the following, simply be referred to as a contract unless a further distinction is necessary.

Definition 5 (Contract). A contract $\mathcal{C}$ is a pair $(\mathcal{A}, \mathrm{G})$, where

i) $\mathrm{G}$ is an assertion, called guarantee; and

ii) $\mathcal{A}$ is a set of assertions $\left\{\mathrm{A}_{i}\right\}_{i=1}^{N}$ where each $\mathrm{A}_{i}$ is called an assumption. 
The set of variables over which an assumption $A_{i} \in \mathcal{A}$ or a guarantee $\mathrm{G}$ is expressed, is called the scope of $\mathrm{A}_{i}$ or $\mathrm{G}$, denoted $X_{\mathrm{A}_{i}}$ and $X_{\mathrm{G}}$, respectively. For the sake of readability, let $\mathrm{A}_{\mathcal{A}}=\widehat{\bigcap}_{j=1}^{N} \mathrm{~A}_{i}$ and $X_{\mathrm{A}_{\mathfrak{A}}}=\bigcup_{i=1}^{N} X_{\mathrm{A}_{i}}$.

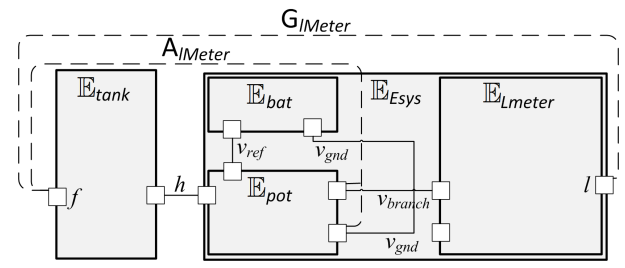

Fig. 3. A contract $\mathcal{C}_{l \text { Meter }}=\left(\left\{\mathrm{A}_{\text {lMeter }}\right\}, \mathrm{G}_{l \text { Meter }}\right)$.

As an illustrative example, consider the architecture $\mathscr{A}_{L M s y s}$ as shown in Fig. 2 and a contract $\mathcal{C}_{l \text { Meter }}=\left(\left\{\mathrm{A}_{l \text { Meter }}\right\}, \mathrm{G}_{\text {lMeter }}\right)$ for the level meter $\mathbb{E}_{l \text { Meter }}$ as shown in Fig. 3 where the dashed lines represent the scopes of $\mathrm{A}_{l \text { Meter }}$ and $\mathrm{G}_{l \text { Meter }}$, respectively. The assertion $\mathrm{G}_{l \text { Meter }}$, specified by the equation $l=f$, expresses that the responsibility of $\mathbb{E}_{l \text { Meter }}$ is to ensure that the presented fuel level $l$, shown by the meter, shall correspond to the level $f$ in the tank. However, in order for the level meter $\mathbb{E}_{l \text { Meter }}$ to be able to ensure that $G_{l \text { Meter }}$ holds, the voltage measured between $v_{\text {branch }}$ and $v_{\text {gnd }}$ on $\mathbb{E}_{l M e t e r}$ must map to a specific level in the tank. That is, the assumption $\mathrm{A}_{l \text { Meter }}$ is specified by the equation $f=\frac{v_{\text {branch }}-v_{\text {gnd }}}{5}$. $\mathrm{G}_{\text {lMeter }}$

While contracts in current contract theories $[1,5-9,11-21]$ are limited to express the intended behavior of $\mathbb{E}_{l \text { Meter }}$ as a relation between the voltage connections and the indicated level, Definition 5 allows assigning the responsibility of achieving the guarantee $G_{l M e t e r}$ to $\mathbb{E}_{l \text { Meter }}$ where $G_{l \text { Meter }}$ has a scope that extends outside of the interface of $\mathbb{E}_{l \text { Meter }}$.

\subsection{Conditions on Element and Environment}

In this section, conditions on an element and the environment of the element are presented, where the conditions ensure that the overall property expressed by the guarantee of a contract is met.

As previously indicated, a guarantee of a contract $\mathcal{C}=(\mathcal{A}, \mathrm{G})$ expresses an intended property under the responsibility of an element $\mathbb{E}$, given that the environment of the element fulfills the assumptions in an architecture $\mathscr{A}$. Formulated differently, it means that the responsibility of the element is to ensure that the overall property expressed by the guarantee is met, i.e. that $B_{E_{n v_{\mathscr{A}}}(\mathbb{E})} \widehat{\cap} \mathrm{B} \widehat{\subseteq} \mathrm{G}$, given that the environment fulfills the assumptions, i.e. that $\mathrm{B}_{E n v_{\mathscr{A}}}(\mathbb{E}) \widehat{\subseteq} \mathrm{A}_{\mathcal{A}}$.

Notably, if it holds that $\mathrm{B}_{E n v_{\mathscr{A}}(\mathbb{E})} \widehat{\subseteq} \mathrm{A}_{\mathcal{A}}$ and $\mathrm{A}_{\mathcal{A}} \widehat{\cap} \mathrm{B} \widehat{\subseteq} \mathrm{G}$, then it follows that $\mathrm{B}_{E n v_{\mathscr{A}}(\mathbb{E})} \widehat{\cap} \mathrm{B} \widehat{\subseteq} \mathrm{G}$. In accordance with $[6-8,20,21], \mathbb{E}$ is said to satisfy $\mathcal{C}$, denoted $\mathbb{E}=\mathcal{C}$, if 


$$
A_{\mathscr{A}} \widehat{\cap} \mathrm{B} \widehat{\subseteq} G \text {. }
$$

Hence, the conditions in order for it to hold that $\mathrm{B}_{\operatorname{Env}_{\mathscr{A}}(\mathbb{E})} \widehat{\cap} \mathrm{B} \widehat{\subseteq} \mathrm{G}$ in a given architecture, can be partitioned into the relation $B_{E n v_{\mathscr{A}}(\mathbb{E})} \widehat{\subseteq} \mathrm{A}_{\mathfrak{A}}$ that expresses a condition on the environment of $\mathbb{E}$, and the relation $A_{\mathcal{A}} \widehat{\cap} \mathrm{B} \widehat{\subseteq} \mathrm{G}$, which is a condition on the element $\mathbb{E}$.

Notably, however, these conditions do not ensure that $\mathrm{B}_{\operatorname{Env}_{\mathscr{S A}}(\mathbb{E})} \hat{\cap} \mathrm{B} \neq \emptyset$, which, according to Proposition 1, implies that the architecture is realizable. Since this is a necessary property in order to develop the product in practice, additional conditions must hence be imposed on the environment and on the element in order to ensure that not only the guarantee holds, but also that the architecture is realizable, i.e. that

$$
\emptyset \subset \mathrm{B}_{E_{n n \mathscr{A}}(\mathbb{E})} \hat{\cap} \mathrm{B} \widehat{\subseteq} \mathrm{G} .
$$

Proposition 2. Consider a contract $\mathcal{C}=(\mathcal{A}, \mathrm{G})$ and an element $\mathbb{E}=(X, \mathrm{~B})$. If $\mathscr{A}$ is an architecture containing $\mathbb{E}$ where $\emptyset \subset \mathrm{B}_{E n v_{\mathscr{A}}(\mathbb{E})} \widehat{\cap} \mathrm{B} \widehat{\subseteq} \mathrm{G}$, then it holds that $\mathrm{B}_{E n v_{\mathscr{A}}(\mathbb{E})} \widehat{\cap} \mathrm{G} \neq \emptyset$.

Proof. Follows from Lemma 8.

Proposition 2 expresses the necessary condition $B_{E n v_{\mathscr{A}}(\mathbb{E})} \widehat{\cap} G \neq \emptyset$ for the relation (14), which means that it is hence not enough to simply require that the environment fulfills the assumptions in order for the relation (14) to hold. Now that this necessary condition on the environment has been identified, the following theorem expresses a complementary condition on the element in order to ensure that the relation (14) holds.

Theorem 1. Consider a contract $\mathcal{C}=(\mathcal{A}, \mathrm{G})$ and an element $\mathbb{E}=(X, \mathrm{~B})$ where $\mathrm{A}_{\mathcal{A}} \widehat{\cap} \mathrm{B} \widehat{\subseteq} \mathrm{G}$. It holds that $\mathrm{A}_{\mathcal{A}} \widehat{\cap} \mathrm{G} \widehat{\subseteq} \mathrm{B}$, if and only if $\emptyset \subset \mathrm{B} \widehat{\cap} \mathrm{B}_{E n v_{\mathscr{A}}}(\mathbb{E}) \widehat{\subseteq} \mathrm{G}$ for each architecture $\mathscr{A}$ containing $\mathbb{E}$ where $\mathrm{B}_{E n v_{\mathscr{A}}(\mathbb{E})} \widehat{\cap} \mathrm{G} \neq \emptyset$ and $\mathrm{B}_{E n v_{\mathscr{A}}(\mathbb{E})} \widehat{\subseteq} \mathrm{A}_{\mathscr{A}}$.

Proof. For the if-only part, consider a contract $\mathcal{C}=(\mathcal{A}, \mathrm{G})$ and an element $\mathbb{E}=(X, \mathrm{~B})$ where

$$
\mathbb{E} \models \mathcal{C} .
$$

Assume that

$$
\mathrm{A}_{\mathscr{A}} \widehat{\cap} \mathrm{G} \widehat{\subseteq} \mathrm{B} .
$$

Furthermore, consider an architecture $\mathscr{A}$ containing $\mathbb{E}$ where

$$
\begin{aligned}
& \mathrm{B}_{\operatorname{Env}_{\mathscr{A}}(\mathbb{E})} \hat{\subseteq} \mathrm{A}_{\mathfrak{A}} \text {, and } \\
& \mathrm{B}_{\operatorname{Env}_{\mathscr{A}}(\mathbb{E})} \hat{\cap} \mathrm{G} \neq \emptyset .
\end{aligned}
$$


The relations (16) and (17), and Lemma 4 imply that

$$
\mathrm{B}_{\operatorname{Env}_{\mathscr{A}}(\mathbb{E})} \widehat{\cap} \mathrm{G} \widehat{\subseteq} \mathrm{B} .
$$

This and the relation (18) imply that

$$
\emptyset \subset \mathrm{B}_{E_{n v_{\mathscr{A}}}(\mathbb{E})} \widehat{\cap} \mathrm{G} \widehat{\subseteq} \mathrm{B} .
$$

This and Lemma 8 imply that

$$
\mathrm{B}_{\operatorname{Env}_{\mathscr{A}}(\mathbb{E})} \widehat{\cap} \mathrm{B} \neq \emptyset .
$$

In accordance with the relation 13, the relation (15) means that

$$
A_{\mathcal{A}} \widehat{\cap} \mathrm{B} \widehat{\subseteq} \mathrm{G}
$$

This, the relation (17), and Lemma 4 imply that

$$
\mathrm{B}_{\operatorname{Env}_{\mathscr{A}}(\mathbb{E})} \widehat{\cap} \mathrm{B} \widehat{\subseteq} \mathrm{G} .
$$

This and the relation (21) imply that

$$
\emptyset \subset \mathrm{B} \widehat{\cap} \mathrm{B}_{E n v_{\mathscr{A}}(\mathbb{E})} \widehat{\subseteq} \mathrm{G} .
$$

Since $\mathscr{A}$ is an arbitrary architecture where the relations (17) and (18) hold, it must hold that the relation (23) also holds for each architecture where the relation (17) holds. This completes the if-only part of the proof. where

For the if part, consider a contract $\mathcal{C}=(\mathcal{A}, \mathrm{G})$ and an element $\mathbb{E}=(X, \mathrm{~B})$

$$
\mathbb{E} \models \mathcal{C}
$$

Assume that for each architecture containing $\mathbb{E}$ where the relations (17) and (18) hold, it follows that

$$
\emptyset \subset \mathrm{B}_{E_{n v_{\mathscr{A}}}(\mathbb{E})} \widehat{\cap} \mathrm{B} \widehat{\subseteq} \mathrm{G} .
$$

In accordance with the relation 13, the relation (24) means that

$$
A_{\mathcal{A}} \widehat{\cap} \mathrm{B} \widehat{\subseteq} \mathrm{G} .
$$

Assume the contradictory relation

$$
\mathrm{A}_{\mathcal{A}} \widehat{\cap} \mathrm{G} \widehat{\nsubseteq} \mathrm{B} \text {. }
$$

This means that there exists a run $\omega$ such that

$$
\{\omega\} \widehat{\subseteq} A_{\mathcal{A}} \widehat{\cap} \mathrm{G} \text { and }\{\omega\} \widehat{\not \subseteq} \mathrm{B} \text {. }
$$

Assume that there exists an architecture $\mathscr{A}$ containing $\mathbb{E}$ where $B_{E_{n v_{\mathscr{A}}(\mathbb{E})}}=$ $\{\omega\}$. This and the relation (28) imply that $\mathscr{A}$ is an architecture where both the 
relations (17) and (18) hold. According to the relation (25), this means that it follows that

$$
\emptyset \subset \mathrm{B}_{\operatorname{Env}_{\mathscr{A}}(\mathbb{E})} \widehat{\cap} \mathrm{B} \widehat{\subseteq} \mathrm{G} .
$$

This is however a contradiction since the relation (28) implies that

$$
\mathrm{B}_{\operatorname{Env}_{\mathscr{A}}(\mathbb{E})} \widehat{\cap} \mathrm{B}=\emptyset .
$$

It hence follows that the relation (27) cannot be true, and hence that it must hold that $A_{\mathscr{A}} \widehat{\cap} \mathrm{G} \widehat{\subseteq} \mathrm{B}$, which concludes the proof.

Given an element $\mathbb{E}$ that satisfies a contract $\mathcal{C}=(\mathcal{A}, \mathrm{G})$, Theorem 1 expresses the necessary and sufficient condition $A_{\mathcal{A}} \widehat{\cap} \mathrm{G} \widehat{\subseteq} \mathrm{B}$ in order for the relation (14) to hold for each architecture $\mathscr{A}$ where $\mathrm{B}_{E n v_{\mathscr{A}}(\mathbb{E})} \widehat{\cap} \mathrm{G} \neq \emptyset$ and $\mathrm{B}_{E n v_{\mathscr{A}}(\mathbb{E})} \widehat{\subseteq} \mathrm{A}_{\mathscr{A}}$.

Corollary 1. Given a contract $\mathcal{C}=(\mathcal{A}, \mathrm{G})$ and an architecture $\mathscr{A}$ containing an element $\mathbb{E}=(X, \mathrm{~B})$, it holds that $\emptyset \subset \mathrm{B} \widehat{\cap} \mathrm{B}_{E n v_{\mathscr{A}}(\mathbb{E})} \widehat{\subseteq} \mathrm{G}$ if

i) $\mathrm{A}_{\mathcal{A}} \widehat{\cap} \mathrm{B} \widehat{\subseteq} \mathrm{G}$ and $\mathrm{A}_{\mathcal{A}} \widehat{\cap} \mathrm{G} \widehat{\subseteq} \mathrm{B}$, and

$$
\text { ii) } \mathrm{B}_{E n v_{\mathscr{A}}(\mathbb{E})} \subseteq \mathrm{A}_{\mathcal{A}} \text { and } \mathrm{B}_{E n v_{\mathscr{A}}(\mathbb{E})} \widehat{\cap} \mathrm{G} \neq \emptyset \text {. }
$$

The proof for Corollary 1 trivially follows from Theorem 1. For practical application, Corollary 1 cleanly separates the respective conditions that an element $\mathbb{E}$ and the environment of $\mathbb{E}$ need to meet with respect to a contract $\mathcal{C}$, in order to obtain a realizable architecture where the guarantee of $\mathcal{C}$ holds.

\section{$3.2 \quad$ Scoping}

This section presents necessary constraints on the structural properties of a contract in order for the conditions (i) and (ii) of Corollary 1 to hold. The constraints serve as sanity checks in order to determine that a contract is not an unreasonable specification for an element in an architecture.

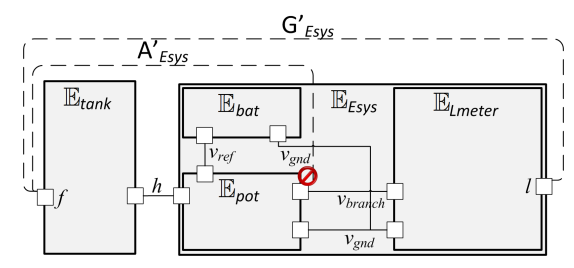

(a)

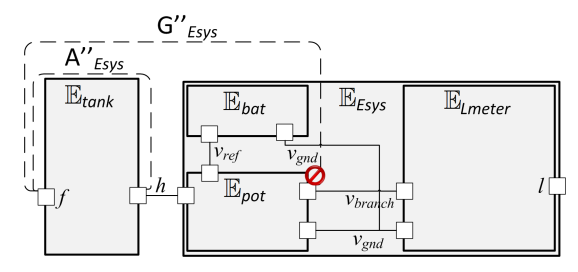

(b)

Fig. 4. Two contracts $\mathcal{C}_{\text {Esys }}^{\prime}$ and $\mathcal{C}_{\text {Esys }}^{\prime \prime}$ for $\mathbb{E}_{\text {Esys }}$ where the conditions (i) and (ii) of Corollary 1 do not hold in the generic case.

Prior to presenting such constraints in a formal manner, two representative examples (a) and (b) are shown in Fig. 4a and $4 \mathrm{~b}$ where the element $\mathbb{E}_{\text {Esys }}$ 
cannot, in the generic case, meet the conditions (i) and (ii) of Corollary 1 with respect to $\mathcal{C}_{E s y s}^{\prime}=\left(\left\{\mathrm{A}_{E s y s}^{\prime}\right\}, \mathrm{G}_{E s y s}^{\prime}\right)$ and $\mathcal{C}_{\text {Esys }}^{\prime \prime}=\left(\left\{\mathrm{A}_{E s y s}^{\prime \prime}\right\}, \mathrm{G}_{E s y s}^{\prime \prime}\right)$, respectively, in the context of the architecture $\mathscr{A}_{L M s y s}$ as shown in Fig 2.

As shown in the examples (a) and (b), since vbranch is in both $\mathrm{A}_{E s y s}^{\prime}$ and $\mathrm{G}_{\text {Esys }}^{\prime \prime}$, but neither in the interface of an element in the environment of $\mathbb{E}_{\text {Esys }}$ nor on the interface of $\mathbb{E}_{E s y s}$, the environment of $\mathbb{E}_{E s y s}$ cannot fulfill $\mathrm{A}_{E s y s}^{\prime}$ and $\mathbb{E}_{E s y s}$ cannot satisfy $\mathcal{C}_{\text {Esys }}^{\prime \prime}$ in the generic case. This hence means that $\mathbb{E}_{\text {Esys }}$ and $\operatorname{Env}_{\mathscr{A}_{L M s y s}}\left(\mathbb{E}_{\text {Esys }}\right)$ cannot meet the respective conditions (i) and (i) of Corollary 1 with respect to neither $\mathcal{C}_{\text {Esys }}^{\prime}$ nor $\mathcal{C}_{\text {Esys }}^{\prime \prime}$.

Theorem 2. Given a contract $\mathcal{C}=(\mathcal{A}, \mathrm{G})$, if $\mathscr{A}$ is an architecture containing

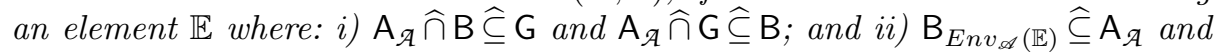
$\mathrm{B}_{E n v_{\mathscr{A}}(\mathbb{E})} \hat{\cap} \mathrm{G} \neq \emptyset$, then it holds that

a) $X_{A_{g}}^{\prime} \subseteq X_{E n v_{\mathscr{A}}(\mathbb{E})}$; and

b) $X_{\mathrm{G}}^{\prime} \subseteq X_{E n v_{\mathscr{A}}(\mathbb{E})} \cup X$,

where $X_{\mathrm{A}_{\mathfrak{A}}}^{\prime} \subseteq X_{\mathrm{A}_{\mathscr{A}}}$ and $X_{\mathrm{G}}^{\prime} \subseteq X_{\mathrm{G}}$ are the sets of variables constrained by $\mathrm{A}_{\mathscr{A}}$ and $\mathrm{G}$, respectively, and $X_{E n v_{\mathscr{A}}(\mathbb{E})}$ denotes the union of the interfaces of the elements in the environment of $\mathbb{E}$ in $\mathscr{A}$.

Proof. Given a contract $\mathcal{C}=(\mathcal{A}, \mathrm{G})$, assume that there exists an architecture $\mathscr{A}$ containing an element $\mathbb{E}$ where the conditions (i) and (ii) of Theorem 2 hold. This and Corollary 1 imply that

$$
\emptyset \subset \mathrm{B} \widehat{\cap} \mathrm{B}_{E_{n v_{\mathscr{A}}}(\mathbb{E})} \widehat{\widehat{C} \mathrm{G}} .
$$

Since $\mathrm{B}$ is over $X$ and $\mathrm{B}_{E n v_{\mathscr{A}}(\mathbb{E})}$ is over $X_{E n v_{\mathscr{A}}(\mathbb{E})}$, Lemma 3 implies that $\mathrm{B} \widehat{\cap} \mathrm{B}_{E n v_{\mathscr{A}}(\mathbb{E})}$ can only constrain a subset of $X_{E n v_{\mathscr{A}}(\mathbb{E})} \cup X$. This and Lemma 9 imply that the set of variables $X_{\mathrm{G}}^{\prime}$ constrained by G is a subset of $X_{E n v_{\mathscr{A}}(\mathbb{E})} \cup X$, i.e. that

$$
X_{\mathrm{G}}^{\prime} \subseteq X_{\operatorname{Env}_{\mathscr{A}}(\mathbb{E})} \cup X .
$$

Since $\mathrm{B}_{E n v_{\mathscr{A}}(\mathbb{E})}$ is over $X_{E n v_{\mathscr{A}}(\mathbb{E})}$, Lemma 3 implies that $\mathrm{B}_{E n v_{\mathscr{A}}(\mathbb{E})}$ can only constrain a subset of $X_{E n v_{\mathscr{A}}(\mathbb{E})}$. This and due to the fact that condition (ii) of Theorem 2 implies that $\mathrm{B}_{E n v_{\mathscr{A}}(\mathbb{E})} \widehat{\subseteq} \mathrm{A}_{\mathfrak{A}}$, in accordance with Lemma 9, it holds that the set of variables $X_{\mathrm{A}_{\mathscr{A}}}^{\prime}$ constrained by $\mathrm{A}_{\mathscr{A}}$ is a subset of $X_{E n v_{\mathscr{A}}(\mathbb{E})}$, i.e. that

$$
X_{\mathrm{A}_{\mathfrak{A}}}^{\prime} \subseteq X_{\operatorname{Env}_{\mathscr{A}}(\mathbb{E})} .
$$

This and the relation (30) concludes the proof.

The relations (a) and (b) of Theorem 2 express necessary conditions on the structural properties of a contract $\mathcal{C}=(\mathcal{A}, \mathrm{G})$ in order for an element and its environment to meet the conditions (i) and (ii) of Corollary 1 in the context of an architecture. Considering Theorem 2, a contract $\mathcal{C}$ is said to be scope-compliant for $\mathbb{E}=(X, \mathrm{~B})$ in an architecture $\mathscr{A}$, if the respective scopes of $\mathrm{A}_{\mathscr{A}}$ and $\mathrm{G}$ are subsets of $X_{E n v_{\mathscr{A}}(\mathbb{E})}$ and $X_{E n v_{\mathscr{A}}(\mathbb{E})} \cup X$. 
Definition 6 (Scope-Compliant Contract for Element). A contract ( $\mathcal{A}, \mathrm{G})$ is scope-compliant for an element $\mathbb{E}=(X, \mathrm{~B})$ in an architecture $\mathscr{A}$, if

a) $X_{\mathrm{A}_{\mathscr{A}}} \subseteq X_{E n v_{\mathscr{A}}(\mathbb{E})}$; and

b) $X_{\mathrm{G}} \subseteq X_{E n v_{\mathscr{A}}(\mathbb{E})} \cup X$.

Under the assumption that the scopes of $A_{\mathcal{A}}$ and $G$ are equal to the set of variables which they constrain, relations (a) and (b) of Definition 6 hold for all cases where the relations (i) and (ii) of Theorem 2 also hold. This includes all of the practical cases since the relations (i) and (ii) of Theorem 2 still hold regardless of the inclusion of all variables that are not constrained by $A_{\mathcal{A}}$ and $G$ in $X_{\mathrm{A}_{\mathfrak{A}}}$ and $X_{\mathrm{G}}$, respectively.

Regarding the examples shown in Fig. $4 \mathrm{a}$ and $4 \mathrm{~b}$, since $X_{\mathrm{A}_{E s y s}^{\prime}} \not X_{\text {tank }}$, in accordance with relation (a) of Definition 6, the contract $\mathcal{C}_{E s y s}^{\prime}$ is not scopecompliant for $\mathbb{E}_{E s y s}$ in $\mathscr{A}_{\text {LMsys }}$. Furthermore, since $X_{\mathrm{G}_{E s y s}^{\prime \prime}} \nsubseteq X_{\text {tank }} \cup X_{\text {Esys }}$, in accordance with relation (b) of Definition 6, the contract $\mathcal{C}_{\text {Esys }}^{\prime \prime}$ is not scopecompliant for $\mathbb{E}_{\text {Esys }}$ in $\mathscr{A}_{\text {LMsys }}$. Definition 6 hence provides a means to detect that both the contracts $\mathcal{C}_{\text {Esys }}^{\prime}$ and $\mathcal{C}_{\text {Esys }}^{\prime \prime}$ are unreasonable specifications for $\mathbb{E}_{\text {Esys }}$ in $\mathscr{A}_{\text {LMsys }}$ considering the scopes of the assumptions and the guarantees.

\section{Properties of Environment-Centric Contracts}

Since contracts in current contract theories $[1,5-9,11-21]$ are limited to element interfaces, definitions that specify whether a contract or a set of contracts has a certain property or not, are also limited to the cases where contracts are limited to the interfaces of elements. Hence, in order to support the use of environmentcentric contracts, the properties of contracts need to be revised, and subsequently proven to be valid.

This section presents revised definitions of the properties consistency $[6,7,20]$, compatibility $[6,7,20]$, and dominance $[8,21]$ of contracts as defined in current contract theories, as well as conditions for when such properties hold.

\subsection{Consistency and Compatibility}

In order to get a better understanding of when the properties consistency $[6,7,20]$ and compatibility $[6,7,20]$ are relevant, a scenario is examined where a contract is used to out source the development of an element $\mathbb{E}$ with an interface $X$. Specifically, the scenario can be described in three phases:

1) a contract $(\mathcal{A}, G)$ and an interface specification $X$ are handed from the client to a supplier;

2) an element $\mathbb{E}=(X, \mathrm{~B})$ is delivered to the client that meets the condition (i) of Corollary 1 ; and

3) the client integrates the element $\mathbb{E}$ with a set of elements to form an architecture where the environment of $\mathbb{E}$ meets the condition (ii) of Corollary 1. 
As expressed in phases (1-2), the client would expect the supplier to deliver an element that meets the condition (i) of Corollary 1 with respect to the contract $\mathcal{C}$. However, in order for the supplier to be able to meet the demands from the client, the supplier would expect that the contract is such that there actually exists an element that meets the condition (i) of Corollary 1. If such an element exists, then the contract will be referred to as a consistent contract.

Furthermore, in order for the client to be able to complete phase (3), at least one architecture containing an element $\mathbb{E}=(X, \mathrm{~B})$ where the environment of $\mathbb{E}$ meets the condition (ii) of Corollary 1 , needs to exist. If such an architecture exists, then the contract will be referred to as a compatible contract.

Now that the concepts of consistency and compatibility have been introduced in the context of a scenario, formal definitions follow.

Definition 7 (Consistent Contract). A contract $(\mathcal{A}, \mathrm{G})$ is consistent with respect to a set of variables $X$ if there exists an element $\mathbb{E}=(X, \mathrm{~B})$ such that $\mathrm{A}_{\mathcal{A}} \widehat{\cap} \mathrm{B} \widehat{\subseteq} \mathrm{G}$ and $\mathrm{A}_{\mathcal{A}} \widehat{\cap} \mathrm{G} \widehat{\subseteq} \mathrm{B}$.

Definition 7 is essentially a revision of the definition of consistency in [20] by considering the condition (i) of Corollary 4.1. Definition 7 is also closely related to definitions in $[6,7]$, but where Definition 7 , in contrast to the definitions in $[6,7]$, allows contracts that are not limited to the interface and are further not limited to elements with defined inputs and outputs.

Theorem 3. A contract $(\mathcal{A}, \mathrm{G})$ is consistent with respect to a set of variables $X$, if and only if $\mathrm{A}_{\mathcal{A}} \widehat{\cap} \widehat{\operatorname{proj}_{X}}\left(\mathrm{~A}_{\mathcal{A}} \widehat{\cap} \mathrm{G}\right) \widehat{\subseteq} \mathrm{G}$.

Proof. For the only-if part, assume that a contract $\mathcal{C}=(\mathcal{A}, \mathrm{G})$ is consistent with respect to a set of variables $X$. In accordance with Definition 7, this means that there exists an element $\mathbb{E}=(X, \mathrm{~B})$ such that

$$
\begin{aligned}
& \mathbb{E} \models \mathcal{C} \text {, and } \\
& \mathrm{A}_{\mathcal{A}} \widehat{\cap} \mathrm{G} \widehat{\subseteq} \mathrm{B} .
\end{aligned}
$$

In accordance with the relation 13, the relation (31) means that

$$
\mathrm{A}_{\mathcal{A}} \widehat{\cap} \mathrm{B} \widehat{\subseteq} \mathrm{G} .
$$

The relation (32) and Lemma 2 imply that

$$
\widehat{\operatorname{proj}_{X}}\left(\mathrm{~A}_{\mathcal{A}} \widehat{\cap} \mathrm{G}\right) \widehat{\subseteq} \widehat{\operatorname{proj}}_{X}(\mathrm{~B}) \text {. }
$$

This, Lemma 1 , and since $B$ is an assertion over $X$ imply that

$$
\widehat{\operatorname{proj}}_{X}\left(\mathrm{~A}_{\mathcal{A}} \widehat{\cap} \mathrm{G}\right) \widehat{\subseteq} \mathrm{B} .
$$

This, the relation (33), and Lemma 4 imply that

$$
\mathrm{A}_{\mathcal{A}} \widehat{\cap} \widehat{\operatorname{proj}}_{X}\left(\mathrm{~A}_{\mathcal{A}} \widehat{\cap} \mathrm{G}\right) \widehat{\subseteq} \mathrm{G} .
$$


This means that the if-only part has been proven and the following will hence concern the if part.

Given a contract $\mathcal{C}=(\mathcal{A}, \mathrm{G})$ and a set of variables $X$, assume that the relation (36) holds. Let $\mathbb{E}=(X, \mathrm{~B})$ be an element where

$$
\mathrm{B}=\widehat{\operatorname{proj}_{X}}\left(\mathrm{~A}_{\mathcal{A}} \widehat{\cap} \mathrm{G}\right) .
$$

This and the relation (36) imply that

$$
A_{\mathcal{A}} \widehat{\cap} \mathrm{B} \widehat{\subseteq} \mathrm{G} .
$$

Lemma 5 implies that

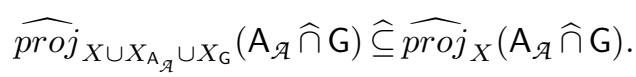

In accordance with Sec. 2.1, this can also be written as

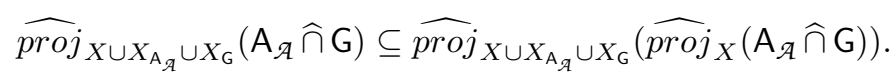

Again, in accordance with Sec. 2.1, this can also be written as

$$
\mathrm{A}_{\mathcal{A}} \widehat{\cap} \mathrm{G} \widehat{\subseteq} \widehat{\operatorname{proj}} j_{X}\left(\mathrm{~A}_{\mathcal{A}} \widehat{\cap} \mathrm{G}\right) .
$$

This and the relation (37) imply that

$$
A_{\mathcal{A}} \widehat{\cap} \mathrm{G} \widehat{\subseteq} B .
$$

This and the relation (38) completes the proof.

Given a contract $\mathcal{C}=(\mathcal{A}, G)$, Theorem 3 supports a way of verifying that $\mathcal{C}$ is consistent with respect to $X$ or not, without having to go through all possible elements with an interface $X$ in order to determine whether there exists an element $\mathbb{E}=(X, \mathrm{~B})$ that meets the conditions (i) of Corollary 1.

Corollary 2. Given a consistent contract $\mathcal{C}=(\mathcal{A}, \mathrm{G})$ with respect to a set of variables $X$, it holds that $X_{\mathrm{G}}^{\prime} \subseteq X_{\mathrm{A}_{\mathfrak{A}}} \cup X$, where $X_{\mathrm{G}}^{\prime} \subseteq X_{\mathrm{G}}$ is the set of variables constrained by $\mathrm{G}$.

Proof. Consider a consistent contract $\mathcal{C}=(\mathcal{A}, \mathrm{G})$ with respect to a set of variables $X$. This and Theorem 3 imply that

$$
\mathrm{A}_{\mathcal{A}} \widehat{\cap} \widehat{\operatorname{proj}}_{X}\left(\mathrm{~A}_{\mathcal{A}} \widehat{\cap} \mathrm{G}\right) \widehat{\subseteq} \mathrm{G} .
$$

Since $\mathrm{A}_{\mathcal{A}} \widehat{\cap} \widehat{\operatorname{proj}}{ }_{X}\left(\mathrm{~A}_{\mathcal{A}} \widehat{\cap} \mathrm{G}\right)$ is an assertion over $X \cup X_{\mathrm{A}_{\mathscr{A}}}$, Lemma 3 implies that $\mathrm{A}_{\mathcal{A}} \widehat{\cap} \widehat{\operatorname{proj}}{ }_{X}\left(\mathrm{~A}_{\mathcal{A}} \widehat{\cap} \mathrm{G}\right)$ can only constrain a subset of $X_{\mathrm{A}_{\mathfrak{A}}} \cup X$. This, the relation (39) and Lemma 9 imply that

$$
X_{\mathrm{G}}^{\prime} \subseteq X_{\mathrm{A}_{\mathfrak{A}}} \cup X
$$

where $X_{\mathrm{G}}^{\prime} \subseteq X_{\mathrm{G}}$ is the set of variables constrained by $\mathrm{G}$. 
Similar to Theorem 2, Corollary 2 expresses a structural property of $\mathcal{C}$ that constitutes a necessary condition in order for $\mathcal{C}$ to be consistent with respect to $X$. In the generic case, i.e. when $X_{\mathrm{G}}^{\prime}=X_{\mathrm{G}}$, the scope of $\mathrm{G}$ must be a subset of $X_{\mathrm{A}_{\mathfrak{A}}} \cup X$.

Definition 8 (Compatible Contract). A contract $(\mathcal{A}, \mathrm{G})$ is compatible with respect to a set of variables $X$ if there exists an architecture $\mathscr{A}$ containing an element $\mathbb{E}=(X, \mathrm{~B})$, such that $\mathrm{B}_{E n v_{\mathscr{A}}(\mathbb{E})} \widehat{\cap} \mathrm{G} \neq \emptyset$ and $\mathrm{B}_{E n v_{\mathscr{A}}(\mathbb{E})} \widehat{\subseteq} \mathrm{A}_{\mathcal{A}}$.

Definition 8 is essentially a revision of the definitions of compatibility in [20] by considering the condition (ii) of Corollary 4.1. Definition 8 is also closely related to the definitions of compatibility in $[6,7]$, but where Definition 8 , in contrast to the definitions in $[6,7]$, allows contracts that are not limited to the interface and are further not limited to elements with causal behaviors, i.e. with defined inputs and outputs.

Theorem 4. A contract $(\mathcal{A}, \mathrm{G})$ is compatible with respect to a set of variables $X$ if and only if $\mathrm{A}_{\mathcal{A}} \widehat{\cap} \mathrm{G} \neq \emptyset$.

Proof. For the if part, consider a contract $(\mathcal{A}, \mathrm{G})$ and assume that it holds that $\mathrm{A}_{\mathcal{A}} \widehat{\cap} \mathrm{G} \neq \emptyset$. In accordance with Sec. 2.1, this can also be written as

$$
\widehat{\operatorname{proj}} X_{X_{\mathrm{A}_{\mathcal{A}}} \cup X_{\mathrm{G}}}\left(\mathrm{A}_{\mathcal{A}}\right) \cap \widehat{\operatorname{proj}}{X_{\mathrm{A}_{\mathcal{H}}} \cup X_{\mathrm{G}}}(\mathrm{G}) \neq \emptyset
$$

This implies that there exists a run $\omega_{X_{\mathrm{A}_{\mathfrak{A}}}}$ such that

$$
\omega_{X_{\mathrm{A}_{\mathcal{A}}}} \in \mathrm{A}_{\mathcal{A}} \text { and }\left\{\omega_{X_{\mathrm{A}_{\mathcal{H}}}}\right\} \widehat{\subseteq} \mathrm{A}_{\mathcal{A}} \widehat{\cap} \mathrm{G}
$$

Let $\mathscr{A}_{0}$ be an architecture containing an element $\mathbb{E}=(X, \mathrm{~B})$ such that $\mathrm{B}_{E_{n v_{\mathscr{A}_{0}}}(\mathbb{E})}=\left\{\omega_{X_{\mathrm{A}_{\mathcal{A}}}}\right\}$. This and the relation (41) imply that there exists an architecture $\mathscr{A}$ containing an element $\mathbb{E}=(X, \mathrm{~B})$, such that the relations (i) and (ii) of Definition 8 hold. In accordance with Definition 8 , this means that the contract $(\mathcal{A}, \mathrm{G})$ is compatible with respect to $X$.

For the if-only part, assume that a contract $(\mathcal{A}, \mathrm{G})$ is compatible with respect to $X$. In accordance with Definition 8 , this means that there exists an

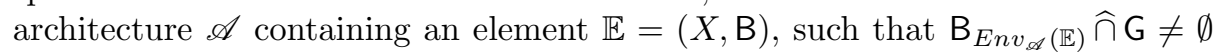
and $\mathrm{B}_{E n v_{\mathscr{A}}(\mathbb{E})} \widehat{\subseteq} \mathrm{A}_{\mathcal{A}}$. This and Lemma 7 imply that $\mathrm{A}_{\mathcal{A}} \widehat{\cap} \mathrm{G} \neq \emptyset$, which completes the proof.

Given a contract $(\mathcal{A}, \mathrm{G})$ and a set of variables $X$, Theorem 4 supports a way of verifying that $(\mathcal{A}, \mathrm{G})$ is compatible with respect to $X$ or not, without having to go through each architecture $\mathscr{A}$ containing an element $\mathbb{E}=(X, \mathrm{~B})$ in order to determine whether there exists an architecture $\mathscr{A}$ where the condition (ii) of Corollary 4.1 hold. 


\subsection{Dominance}

Prior to formally presenting the definition of dominance, a scenario is presented in the context of an Original Equipment Manufacturer (OEM)/supplier chain in order to provide an understanding of when the property is relevant:

1) a contract $\mathcal{C}=(\mathcal{A}, \mathrm{G})$ is decomposed into a set of contracts $\left\{\mathcal{C}_{i}\right\}_{i=1}^{N}$ where each contract $\mathcal{C}_{i}$ and an interface specification $X_{i}$ is handed from the OEM to either a development team within the organization or to a supplier;

2) each development team or supplier develops an element $\mathbb{E}_{i}=\left(X_{i}, \mathrm{~B}_{i}\right)$ that meets the condition (i) of Corollary 1 with respect to $\mathcal{C}_{i}$; and

3) the OEM integrates the set of elements $\mathbb{E}_{i}$ with each other to form an element $\mathbb{E}$ that meets the condition (i) of Corollary 1 with respect to $\mathcal{C}$.

As expressed in phase (3), the overall intent is to obtain an element $\mathbb{E}$ that meets the condition (i) of Corollary 1 with respect to $\mathcal{C}$. In order to achieve this, the intent is hence to decompose the contract $\mathcal{C}$ into the set $\left\{\mathcal{C}_{i}\right\}_{i=1}^{N}$ such that if each $\mathbb{E}_{i}$ meets the condition (i) of Corollary 1 with respect to $\mathcal{C}_{i}$, then $\mathbb{E}$ meets the condition (i) of Corollary 1 with respect to $\mathcal{C}$. If such a property holds, then $\mathcal{C}$ is said to dominate the set of contracts $\left\{\mathcal{C}_{i}\right\}_{i=1}^{N}$.

Definition 9 (Dominance of Contracts). Given a set of variables $X$, a contract $\mathcal{C}$ and a set of contracts $\left\{\mathcal{C}_{i}\right\}_{i=1}^{N}$, the contract $\mathcal{C}$ dominates $\left\{\mathcal{C}_{i}\right\}_{i=1}^{N}$ if for any architecture $\mathscr{A}$ where $\mathcal{C}$ is scope-compliant for an element $\mathbb{E}=(X, \mathrm{~B})$ in $\mathscr{A}$ and where $\left\{\mathbb{E}_{i}=\left(X_{i}, \mathrm{~B}\right)\right\}_{i=1}^{N}$ is the set of children of $\mathbb{E}$, it holds that

$$
\mathrm{A}_{\mathcal{A}_{i}} \hat{\cap} \mathrm{B}_{i} \widehat{\subseteq} \mathrm{G}_{i} \text { and } \mathrm{A}_{\mathcal{H}_{i}} \hat{\cap} \mathrm{G}_{i} \widehat{\subseteq} \mathrm{B}_{i} \text { for each } i \Longrightarrow \mathrm{A}_{\mathcal{A}} \widehat{\cap} \mathrm{B} \widehat{\subseteq} \mathrm{G} \text { and } \mathrm{A}_{\mathcal{A}} \widehat{\cap} \mathrm{G} \hat{\subseteq} \mathrm{B} \text {. }
$$

Definition 9 is essentially a generalization of the definitions of dominance presented in $[8,21]$ by relying on the notion of scope-compliance as presented in Definition 6 and by considering the conditions of Corollary 1 .

Decomposition Structures This section introduces a graph, called a decomposition structure, in order to find a decomposition of a contract $\mathcal{C}$ into a set of contracts $\left\{\mathcal{C}_{i}\right\}_{i=1}^{N}$ to achieve the property as expressed in Definition 9. Prior to presenting the formal definition of a decomposition structure, the concept is introduced informally by structuring a decomposition of a contract $\mathcal{C}_{\text {Esys }}$ into a set of contracts $\left\{\mathcal{C}_{\text {pot }}, \mathcal{C}_{\text {bat }}, \mathcal{C}_{\text {lMeter }}\right\}$. The set of contracts represents the specifications of the parts of an electric-system of an LM-system, e.g. the one shown in Fig. 2.

Consider that the assumptions and the guarantees of each contract in the set $\left\{\mathcal{C}_{\text {Esys }}, \mathcal{C}_{\text {pot }}, \mathcal{C}_{\text {bat }}, \mathcal{C}_{\text {lMeter }}\right\}$ are organized as nodes in a directed graph, as shown in Fig. 5 where the boxes with rounded corners and dashed edges represent an hierarchical structure of contracts. The set of incoming arcs to a guarantee $\mathrm{G}$ from a set of assumptions $\mathcal{A}$, represents that $\mathcal{A}$ and $\mathrm{G}$ are in the same contract, e.g. the $\operatorname{arc}$ from $A_{E s y s}$ to the guarantee $\mathrm{G}_{\text {Esys }}$ represents the contract $\left(\left\{\mathrm{A}_{E s y s}\right\}, \mathrm{G}_{\text {Esys }}\right)$. 


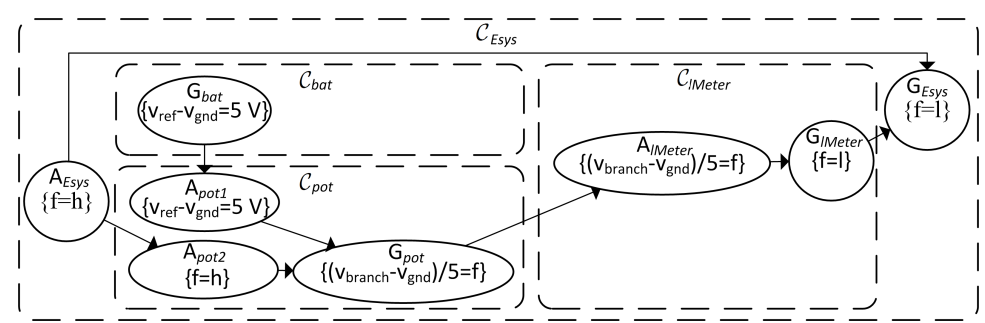

Fig. 5. A decomposition structure of a contract $\mathcal{C}_{\text {Esys }}$ into a set of contracts $\left\{, \mathcal{C}_{\text {pot }}, \mathcal{C}_{\text {bat }}, \mathcal{C}_{\text {lMeter }}\right\}$ where boxes that represent the contracts have been added.

The set of incoming arcs to an assumption $A$ from a set of assertions $\left\{\mathrm{W}_{i}\right\}_{i=1}^{N}$ where $W_{i}$ is either an assumption or a guarantee, represents the intention of $\widehat{\bigcap}_{i=1}^{N} \mathrm{~W}_{i} \widehat{\subseteq} \mathrm{A}$. For example, the arc to $\mathrm{A}_{\text {pot } 2}$ from the assumption $\mathrm{A}_{\text {Esys }}$, represents the intent of $A_{E s y s} \widehat{\subseteq} A_{\text {pot } 2}$. The set of incoming arcs to a guarantee $G$ from a set of guarantees $\left\{\mathrm{G}_{j}\right\}_{j=1}^{M}$ represents the intent of $\widehat{\bigcap}_{j=1}^{M} \mathrm{G}_{j} \widehat{\subseteq} \mathrm{G}$. For example, the arc from the guarantee $G_{l \text { Meter }}$ to $G_{\text {Esys }}$, represents the intent of $G_{l \text { Meter }} \widehat{\subseteq} G_{\text {Esys }}$.

Now that the concept of a decomposition structure has been introduced informally, the formal definition follows.

Definition 10 (Decomposition Structure). $A$ decomposition structure $\mathfrak{D}$ of a contract $(\mathcal{A}, \mathrm{G})$ into a set of contracts $\left\{\left(\mathcal{A}_{i}, \mathrm{G}_{i}\right)\right\}_{i=1}^{N}$ is a Directed Acyclic Graph (DAG), such that:

a) the guarantees $\mathrm{G}_{i}$, the assumptions in each set $\mathcal{A}_{i}$, the assumptions in $\mathcal{A}$, and the guarantee $\mathrm{G}$ are the nodes in $\mathfrak{D}$;

b) $\mathrm{G}$ has no successors and at least one $\mathrm{G}_{i}$ is a direct predecessor of $\mathrm{G}$;

c) $\mathrm{G}_{i}$ is the only direct successor of each assumption in $\mathcal{A}_{i}$; and each assumption in $\mathcal{A}_{i}$ has at least one predecessor;

d) each $\mathrm{G}_{i}$ has a direct successor that it is either an assumption in $\mathcal{A}_{k}$ where $k \neq i$ or $\mathrm{G}$;

e) $\mathrm{G}$ is a direct successor of each assumption in $\mathcal{A}$; and if an assumption in $\mathcal{A}$ has a direct successor that is not $\mathrm{G}$, then it is an assumption in $\mathcal{A}_{i}$.

A decomposition structure represents the intended dependencies between the assumptions and guarantees of a set of contracts.

Sufficient Conditions of Dominance Given that a decomposition structure only represents the intended dependencies between the assumptions and the guarantees, a decomposition structure will be referred to as proper if the intended dependencies are, in fact, true.

Given a decomposition structure $\mathfrak{D}$, let $d \operatorname{Pred}()$ denote a function that takes a node $W$ in $\mathfrak{D}$ as input and returns the set of all nodes that are direct predecessors of $\mathrm{W}$. 
Definition 11 (Proper Decomposition Structure). A decomposition structure of a contract $(\mathcal{A}, \mathrm{G})$ into a set of contracts $\left\{\left(\left\{\mathrm{A}_{i j}\right\}_{j=1}^{M_{i}}, \mathrm{G}_{i}\right)\right\}_{i=1}^{N}$ is proper if:

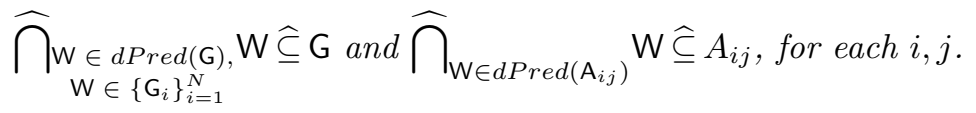

For example, since the relations $G_{b a t} \widehat{\subseteq} A_{\text {pot } 1}, A_{E s y s} \widehat{\subseteq} A_{\text {pot } 2}, G_{p o t} \widehat{\subseteq} A_{l M e t e r}$, and $\mathrm{G}_{\text {lMeter }} \widehat{\widehat{\subseteq}} \mathrm{G}_{\text {Esys }}$ holds in Fig. 5, the decomposition structure is proper according to Definition 11.

However, although a decomposition structure of a contract $(\mathcal{A}, \mathrm{G})$ into a set of contracts $\left\{\left(\mathcal{A}_{i}, \mathrm{G}_{i}\right)\right\}_{i=1}^{N}$ is proper, it does not mean that $(\mathcal{A}, \mathrm{G})$ dominates $\left\{\left(\mathcal{A}_{i}, \mathrm{G}_{i}\right)\right\}_{i=1}^{N}$. The reason for this is that a guarantee $\mathrm{G}_{i}$ can impose harder constraints on a variable than $G$ and this variable can also be constrained by the environment of an element $\mathbb{E}=(X, \mathrm{~B})$ in an architecture $\mathscr{A}$. This means that even if each element $\mathbb{E}_{i}$ meets the condition (i) of Corollary 1 with respect to $\left(\mathcal{A}_{i}, \mathrm{G}_{i}\right)$, it cannot be ensured that it holds that $\mathrm{A}_{\mathcal{A}} \widehat{\cap} \mathrm{G} \widehat{\subseteq} \mathrm{B}$ where $\mathbb{E}$ is the parent of each $\mathbb{E}_{i}$ in $\mathscr{A}$.

Now that the need for yet another condition, in addition to the decomposition being proper, has been introduced, sufficient conditions for dominance now follows.

Theorem 5. Given a set of variables $X$, a contract $\mathcal{C}$ and a set of contracts

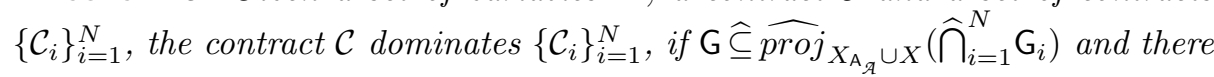
exists a proper decomposition structure of $\mathcal{C}$ into $\left\{\mathcal{C}_{i}\right\}_{i=1}^{N}$.

Proof. Consider a set of variables $X$, a contract $\mathcal{C}$ and a set of contracts $\left\{\mathcal{C}_{i}\right\}_{i=1}^{N}$. Let $\mathfrak{D}$ be a proper decomposition structure of $\mathcal{C}$ into $\left\{\mathcal{C}_{i}\right\}_{i=1}^{N}$ and assume that it holds that

$$
\mathrm{G} \widehat{\subseteq} \widehat{\operatorname{proj}}_{X_{\mathrm{A}} \cup X}\left(\widehat{\bigcap}_{i=1}^{N} \mathrm{G}_{i}\right) .
$$

In accordance with the conditions in Definition 9, assume that there exists an architecture $\mathscr{A}_{0}$ containing an element $\mathbb{E}=(X, \mathrm{~B})$ and a set of elements $\left\{\mathbb{E}_{i}=\left(X_{i}, \mathrm{~B}_{i}\right)\right\}_{i=1}^{N}$ where

$$
\begin{aligned}
& \mathcal{C} \text { is scope-compliant for } \mathbb{E} \text { in } \mathscr{A} \text {, and } \\
& \left\{\mathbb{E}_{i}\right\}_{i=1}^{N} \text { is the set of children of } \mathbb{E} .
\end{aligned}
$$

Assume that, for each $i$,

$$
\begin{aligned}
& \mathbb{E}_{i}=\mathcal{C}_{i} \text {, and } \\
& \mathrm{A}_{\mathcal{A}_{i}} \widehat{\cap} \mathrm{G}_{i} \widehat{\subseteq} \mathrm{B}_{i} .
\end{aligned}
$$

In accordance with the relation 13 , the relation (45) means that 


$$
\mathrm{A}_{\mathcal{A}_{i}} \widehat{\cap} \mathrm{B}_{i} \widehat{\subseteq} \mathrm{G}_{i} \text { for each } i \text {, where } \mathrm{A}_{\mathcal{A}_{i}}=\widehat{\bigcap}_{\mathrm{A}_{i j} \in \mathcal{A}_{i}} \mathrm{~A}_{i j} .
$$

Furthermore, since $\mathfrak{D}$ is a proper decomposition of $\mathcal{C}$ into $\left\{\left(\left\{\mathrm{A}_{i j}\right\}_{j=1}^{M_{i}}, \mathrm{G}_{i}\right)\right\}_{i=1}^{N}$, according to Definition 11, it holds that

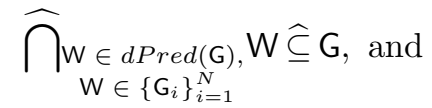

$$
\begin{aligned}
& \widehat{\bigcap}_{\mathrm{W} \in \operatorname{dPred}\left(\mathrm{A}_{i j}\right)} \mathrm{W} \widehat{\subseteq} A_{i j} \text { for each } i, j .
\end{aligned}
$$

Let each $\mathrm{G}_{k}$ in the relation (48) be replaced with $\mathrm{A}_{\mathfrak{A}_{k}} \widehat{\cap} \mathrm{B}_{k}$ in accordance with relation (47) and Lemma 4, and then iteratively, in an alternating fashion, let each assumption $\mathrm{A}_{k j} \in \mathcal{A}_{k}$ be replaced with $\widehat{\bigcap}_{\mathrm{W} \in \operatorname{dPred}\left(\mathrm{A}_{k j}\right)} \mathrm{W}$ in accordance with the relation (49) and Lemma 4 and each guarantee $\mathrm{G}_{u} \in d \operatorname{Pred}\left(\mathrm{A}_{k j}\right)$ with $\mathrm{A}_{\mathcal{A}_{u}} \widehat{\cap} \mathrm{B}_{u}$ in accordance with the relation (47) and Lemma 4 . Since $\mathfrak{D}$ is a Directed Acyclic Graph and each assumption $\mathrm{A}_{i j}$ has a direct predecessor according to Definition 10, it can be inferred that if the relations (49) and (47) are iteratively applied to the relation (48) until there are no further assumptions $\mathrm{A}_{i}$ or guarantees $\mathrm{G}_{i}$, it holds that

$$
\mathrm{A}_{\mathcal{A}} \widehat{\cap}\left(\widehat{\bigcap}_{i=1}^{N} \mathrm{~B}_{i}\right) \widehat{\subseteq} \mathrm{G}
$$

Since each $\mathrm{B}_{i}$ is an assertion over $X_{i}$, it holds that $\widehat{\bigcap}_{i=1}^{N} \mathrm{~B}_{i}$ is an assertion over $\bigcup_{i=1}^{N} X_{i}$. Furthermore, since $\mathcal{C}$ is scope-compliant for $\mathbb{E}$ as stated in the condition (43), any variable $x$ where $x \in \bigcup_{i=1}^{N} X_{i}$ and $x \notin X$, can not be in $X_{\mathrm{A}_{\mathcal{A}}}$ nor $X_{\mathrm{G}}$ according to Definition 6 , which means that

$$
\left(\left(\bigcup_{i=1}^{M} X_{i}\right) \backslash X\right) \cap\left(X_{\mathrm{A}_{\mathfrak{A}}} \cup X_{\mathrm{G}}\right)=\emptyset .
$$

Lemma 10, Lemma 4, and the relations (50) and (51) hence implies that

$$
\mathrm{A}_{\mathcal{A}} \widehat{\cap} \operatorname{proj}_{\left(\bigcup_{i=1}^{N} X_{i}\right) \backslash\left(\left(\cup_{i=1}^{N} X_{i}\right) \backslash X\right)}\left(\widehat{\bigcap}_{i=1}^{N} \mathrm{~B}_{i}\right) \widehat{\subseteq} \mathrm{G} .
$$

Since $\left(\bigcup_{i=1}^{N} X_{i}\right) \backslash\left(\left(\bigcup_{i=1}^{N} X_{i}\right) \backslash X\right)=\left(\bigcup_{i=1}^{N} X_{i}\right) \cap X$, it holds that

$$
\left(\bigcup_{i=1}^{N} X_{i}\right) \backslash\left(\left(\bigcup_{i=1}^{N} X_{i}\right) \backslash X\right) \subseteq X
$$

Lemma 5 and the relations (52) and (53) further implies that

$$
\mathrm{A} \widehat{\cap} \widehat{\operatorname{proj}}_{X}\left(\widehat{\bigcap}_{i=1}^{M} \mathrm{~B}_{i}\right) \widehat{\subseteq} \mathrm{G} .
$$


The relation (53) and since $\mathrm{B}=\widehat{\operatorname{proj}_{X}}\left(\widehat{\bigcap}_{i=1}^{M} \mathrm{~B}_{i}\right)$ according to Definition 2 implies that $\mathrm{A} \widehat{\cap} \mathrm{B} \widehat{\subseteq} \mathrm{G}$. In accordance with the relation 13, it holds that

$$
\mathbb{E} \models \mathcal{C} \text {. }
$$

The relation (47) and Lemma 11 imply that

$$
\widehat{\bigcap}_{i=1}^{N} \mathrm{~A}_{\mathcal{A}_{i}} \widehat{\cap} \widehat{\bigcap}_{i=1}^{N} \mathrm{G}_{i} \widehat{\subseteq} \widehat{\bigcap}_{i=1}^{N} \mathrm{~B}_{i}
$$

This, Lemma 4, and since the relation (49) implies that each assertion $\mathrm{A}_{\mathcal{A}_{i}}$ is fulfilled by a subset of $A_{\mathcal{A}} \widehat{\cap} \widehat{\bigcap}_{i=1}^{N} G_{i}$, means that it holds that

$$
\mathrm{A}_{\mathcal{A}} \widehat{\cap} \widehat{\bigcap}_{i=1}^{N} \mathrm{G}_{i} \widehat{\subseteq} \operatorname{proj}_{X}\left(\widehat{\bigcap}_{i=1}^{N} \mathrm{~B}_{i}\right)
$$

The relation (47) and Lemma 9 and 3 imply that the set of variables $X_{\mathrm{G}_{i}}^{\prime} \subseteq$ $X_{\mathrm{G}_{i}}$ constrained by each guarantee $\mathrm{G}_{i}$ is a subset of $X_{i} \cup X_{\mathrm{A}_{\mathfrak{A}_{i}}}$, i.e. for each $i$,

$$
X_{\mathrm{G}_{i}}^{\prime} \subseteq X_{i} \cup X_{\mathrm{A}_{\mathfrak{A}_{i}}}
$$

The relation (49) and Lemma 9 and 3 imply that set of variables $X_{\mathrm{A}_{\mathcal{A}_{j}}}^{\prime} \subseteq$ $X_{\mathrm{A}_{\mathfrak{A}_{j}}}$ constrained by each assertion $\mathrm{A}_{\mathcal{A}_{j}}$ is a subset of the union of scopes of the assertions that are direct predecessors to $\mathrm{A}_{\mathcal{A}_{j}}$. This and the relation (58) and since $\mathfrak{D}$ is a Directed Acyclic Graph and each assumption $\mathrm{A}_{i j}$ has a direct predecessor according to Definition 10, means that the set of variables $X_{\mathrm{G}_{i}}^{\prime} \subseteq X_{\mathrm{G}_{i}}$ constrained by each guarantee $\mathrm{G}_{i}$ is a subset of $X_{\mathrm{A}_{\mathfrak{A}}} \cup\left(\bigcup_{i=1}^{N} X_{i}\right)$. This means that

$$
\bigcup_{i=1}^{N} X_{\mathrm{G}_{i}}^{\prime} \subseteq X_{\mathrm{A}_{\mathcal{A}}} \cup\left(\bigcup_{i=1}^{N} X_{i}\right)
$$

The relation (57) and since any variable $x$ where $x \in \bigcup_{i=1}^{N} X_{i}$ and $x \notin X$, cannot be in $X_{\mathrm{A}_{\mathfrak{A}}}$ according to Definition 6 , imply, in accordance with Lemma 10, that

$$
\mathrm{A}_{\mathcal{A}} \widehat{\cap} \operatorname{proj}_{\left(\bigcup_{i=1}^{N} X_{G_{i}}\right) \backslash\left(\bigcup_{i=1}^{N} X_{i} \backslash X\right)}\left(\widehat{\bigcap}_{i=1}^{N} \mathrm{G}_{i}\right) \widehat{\subseteq} \operatorname{proj}_{X}\left(\widehat{\bigcap}_{i=1}^{N} \mathrm{~B}_{i}\right) .
$$

This and the relation (59) imply, in accordance with the relation (3), that

$$
\left.\mathrm{A}_{\mathcal{A}} \widehat{\cap} \widehat{\operatorname{proj}} \bigcup_{\left(\bigcup_{i=1}^{N} X_{G_{i}}\right) \backslash\left(\bigcup_{i=1}^{N} X_{i} \backslash X\right)} \widehat{(p r o j}_{X_{\mathcal{A}_{\mathcal{A}}} \cup X}\left(\widehat{\bigcap}_{i=1}^{N} \mathrm{G}_{i}\right)\right) \widehat{\subseteq} \operatorname{proj}_{X}\left(\widehat{\bigcap}_{i=1}^{N} \mathrm{~B}_{i}\right)
$$

This, Lemma 4, and since 


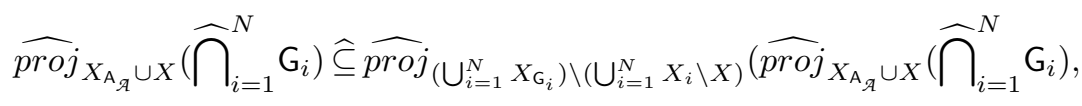

imply that

$$
\mathrm{A}_{\mathcal{A}} \widehat{\cap} \widehat{\operatorname{proj}}_{X_{\mathrm{A}_{\mathcal{A}}} \cup X}\left(\widehat{\bigcap}_{i=1}^{N} \mathrm{G}_{i}\right) \widehat{\subseteq} \operatorname{proj}_{X}\left(\widehat{\bigcap}_{i=1}^{N} \mathrm{~B}_{i}\right)
$$

This and the relations (42) and (44), imply, in accordance with Definition 2 that

$$
A_{\mathcal{A}} \widehat{\cap} \mathrm{G} \widehat{\subseteq} B .
$$

Since $\mathscr{A}_{0}$ is an arbitrary architecture where the relations (43), (44), (45), and (46) hold, the relations (55) and (63) hold for any architecture where the relations (43), (44), (45), and (46) hold. According to Definition 9, this means that $\mathcal{C}$ dominates the set of contracts $\left\{\mathcal{C}_{i}\right\}_{i=1}^{N}$.

The fact that $\mathrm{G} \widehat{\subseteq} \widehat{\operatorname{proj}} X_{\mathrm{A}_{\mathcal{A}} \cup X}\left(\widehat{\bigcap}_{i=1}^{N} \mathrm{G}_{i}\right)$ ensures that the guarantees $\mathrm{G}_{i}$ do not impose harder constraints than $G$ on any variable that can also be constrained by the environment of $\mathbb{E}$ in an architecture.

Considering the decomposition structure of $\mathcal{C}_{\text {Esys }}$ into $\left\{\mathcal{C}_{\text {pot }}, \mathcal{C}_{\text {bat }}, \mathcal{C}_{\text {lMeter }}\right\}$

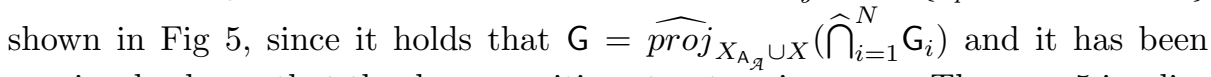
previously shown that the decomposition structure is proper, Theorem 5 implies that $\mathcal{C}_{\text {Esys }}$ dominates $\left\{\mathcal{C}_{\text {pot }}, \mathcal{C}_{\text {bat }}, \mathcal{C}_{\text {lMeter }}\right\}$. Since the contract $\mathcal{C}_{\text {Esys }}$ is scopecompliant for $\mathbb{E}_{\text {Esys }}$ in the architecture shown in Fig. 2 according to Definition 6 , and each child $\mathbb{E}_{i}$ of $\mathbb{E}_{\text {Esys }}$ meets the condition (i) of Corollary 1 with respect to $\mathcal{C}_{i} \in\left\{\mathcal{C}_{\text {pot }}, \mathcal{C}_{\text {bat }}, \mathcal{C}_{\text {lMeter }}\right\}$, in accordance with Definition 9 , it can be inferred that $\mathbb{E}_{\text {Esys }}$ meets the condition (i) of Corollary 1 with respect to $\mathcal{C}_{\text {Esys }}$.

Remark 1 (Circular Reasoning). Since the assumptions and guarantees of a decomposition structure are organized into an acyclic graph, the use of circular argumentation $[13,21,41]$ is avoided.

\section{Conclusion}

As discussed in Sec. 1, in order to be able to express that the responsibility of an element is to achieve an overall intended property of a system, current contract theories need to be generalized to environment-centric contracts. In order to achieve this, a theoretical framework was first introduced in Sec. 2 where the concepts element and architecture were defined in order to model a CPS and its parts.

Building on the theoretical framework, in Sec. 3, the constraint that a contract must be specified over the interface of an element was relaxed and Corollary 1 explicitly declares what conditions an element and an environment need to 
meet, respectively, in order to achieve a realizable architecture where the guarantee holds. Furthermore in Sec. 3, the notion of a scope-compliant contract was introduced that serves as a sanity check that a contract is not an unreasonable specification for an element in an architecture.

Building on Corollary 1, revised definitions of consistency, compatibility, and dominance were presented in Sec. 4. Complementing the definitions, Theorems 3 and 4 express necessary and sufficient conditions of consistency and compatibility, respectively, and Theorem 5 expresses sufficient conditions of dominance based on a graph, called a decomposition structure.

By providing revised definitions of properties of contracts and complementary theorems and definitions for practical application, the present paper fully generalizes current contract theories to environment-centric contracts. As mentioned in Sec. 1, such a generalization provides a much needed support for practical engineering and a necessary capability to properly express safety requirements. 


\section{References}

1. Meyer, B.: Applying "Design by Contract". IEEE Computer 25 (1992) 40-51

2. Misra, J., and K. Chandy: Proofs of networks of processes. Software Engineering, IEEE Transactions on SE-7(4) (1981) 417-426

3. Hoare, C. A. R.: An Axiomatic Basis for Computer Programming. Commun. ACM 12(10) (October 1969) 576-580

4. Dijkstra, E. W.: Guarded Commands, Nondeterminacy and Formal Derivation of Programs. Commun. ACM 18(8) (August 1975) 453-457

5. Jones, C. B.: Specification and Design of (Parallel) Programs. In Mason, R. E. A., ed.: Information Processing 83. Volume 9 of IFIP Congress Series., Paris, France, IFIP, North-Holland (September 1983) 321-332

6. Benveniste, A. et al.: Multiple Viewpoint Contract-Based Specification and Design. In Boer, F. S. et al., eds.: Formal Methods for Components and Object. SpringerVerlag, Berlin, Heidelberg (2008) 200-225

7. Sangiovanni-Vincentelli, A. L., W. Damm, and R. Passerone: Taming Dr. Frankenstein: Contract-Based Design for Cyber-Physical Systems. Eur. J. Control 18(3) (2012) 217-238

8. Bauer, S. et al.: Moving from specifications to contracts in component-based design. In Lara, J., and A. Zisman, eds.: Fundamental Approaches to Software Eng. Volume 7212 of Lec. Notes in Computer Science. Springer Berlin Heidelberg (2012) 43-58

9. Chen, T. et al.: A compositional specification theory for component behaviours. In Seidl, H., ed.: Programming Languages and Systems. Volume 7211 of Lecture Notes in Computer Science. Springer Berlin Heidelberg (2012) 148-168

10. Lee, E.: Cyber Physical Systems: Design Challenges. In: Object Oriented RealTime Distributed Computing (ISORC), 11th IEEE Int. Symp. on. (2008) 363-369

11. Pnueli, A.: Logics and models of concurrent systems. Springer-Verlag New York, Inc., New York, NY, USA (1985) 123-144

12. Shurek, G., and O. Grumberg: The modular framework of computer-aided verification. In Clarke, E., and R. Kurshan, eds.: Computer-Aided Verification. Volume 531 of Lec. Notes in Comp. Science. Springer Berlin Heidelberg (1991) 214-223

13. Abadi, M., and L. Lamport: Composing specifications. ACM Trans. Program. Lang. Syst. 15(1) (January 1993) 73-132

14. Alur, R. et al.: Mocha: Modularity in model checking. In $\mathrm{Hu}, \mathrm{A}$., and M. Vardi, eds.: Computer Aided Verification. Volume 1427 of Lecture Notes in Computer Science. Springer Berlin Heidelberg (1998) 521-525

15. Giese, H.: Contract-based Component System Design. In: Thirty-Third Annual Hawaii Int. Conf. on System Sciences (HICSS-33), Maui, IEEE Press (2000)

16. Sun, X. et al.: Contract-based System-Level Composition of Analog Circuits. In: Design Automation Conf., 2009. DAC '09. 46th ACM/IEEE. (july 2009) 605-610

17. Delahaye, B., B. Caillaud, and A. Legay: Probabilistic contracts: A compositional reasoning methodology for the design of systems with stochastic and/or non-deterministic aspects. Form. Methods Syst. Des. 38(1) (February 2011) 1-32

18. Goesssler, G., D. Xu, and A. Girault: Probabilistic contracts for component-based design. Formal Methods in System Design 41(2) (2012) 211-231

19. Goessler, G., and J.-B. Raclet: Modal contracts for component-based design. In: Proc. of the 2009 7th IEEE Int. Conf. on Software Eng. and Formal Methods. SEFM '09, Washington, DC, USA, IEEE Computer Society (2009) 295-303

20. Benveniste, A. et al.: Contracts for System Design. Rapport de recherche RR-8147, INRIA (November 2012) 
21. Quinton, S., and S. Graf: Contract-based verification of hierarchical systems of components. In: Software Engineering and Formal Methods, 2008. SEFM '08. Sixth IEEE International Conference on. (nov. 2008) $377-381$

22. Chandrasekaran, B., and J. R. Josephson: Function in device representation (2000)

23. Umeda, Y. et al.: Function, behaviour, and structure. Applications of artificial intelligence in engineering V 1 (1990) 177-194

24. Liang, F. et al.: Model-based requirement verification : a case study. Proc. of the 9th Int. Modelica Conf. (2012)

25. Schamai, W. et al.: Towards unified system modeling and simulation with modelicaml: Modeling of executable behavior using graphical notations. In: 7th Modelica Conference 2009, University Electronic Press (2009)

26. Boulanger, J.-L., and V. Q. Dao: Requirements engineering in a model-based methodology for embedded automotive software. In: Research, Innovation and Vision for the Future, 2008. RIVF 2008. IEEE Int. Conf. on. (July 2008) 263-268

27. Friedenthal, S., A. Moore, and R. Steiner: A Practical Guide to SysML: Systems Modeling Language. Morgan Kaufmann Publishers Inc., San Francisco, CA, USA (2008)

28. IEC 61508: Functional safety of electrical/electronic/programmable electronic safety-related systems (2010)

29. ISO 26262: "Road vehicles-Functional safety" (2011)

30. Westman, J., and M. Nyberg: A Reference Example on the Specification of Safety Requirements using ISO 26262. In ROY, M., ed.: Proc. of Workshop DECS of SafeComp, France (September 2013) NA

31. Westman, J., M. Nyberg, and M. Törngren: Structuring Safety Requirements in ISO 26262 Using Contract Theory. In Bitsch, F., J. Guiochet, and M. Kaniche, eds.: Computer Safety, Reliability, and Security. Volume 8153 of Lecture Notes in Computer Science. Springer Berlin Heidelberg (2013) 166-177

32. SPEEDS: SPEculative and Exploratory Design in Sys. Eng. (2006-2009)

33. Codd, E. F.: A Relational Model of Data for Large Shared Data Banks. Commun. ACM 13(6) (June 1970) 377-387

34. ISO/IEC/IEEE 42010: System and software eng. - Architecture description (2011)

35. Păsăreanu, C. S. et al.: Learning to divide and conquer: Applying the $1^{*}$ algorithm to automate assume-guarantee reasoning. Form. Methods Syst. Des. 32(3) (June 2008) 175-205

36. Cobleigh, J. M., G. S. Avrunin, and L. A. Clarke: Breaking up is hard to do: An evaluation of automated assume-guarantee reasoning. ACM Trans. Softw. Eng. Methodol. 17(2) (May 2008) 7:1-7:52

37. Back, R.-J., and J. V. Wright: Contracts, Games and Refinement. In: Information and Computation, Elsevier (1997) 200-0

38. Dill, D. L.: Trace Theory for Automatic Hierarchical Verification of SpeedIndependent Circuits. In: Proceedings of the fifth MIT conference on Advanced research in VLSI, Cambridge, MA, USA, MIT Press (1988) 51-65

39. Alfaro, L. D., and T. A. Henzinger: Interface Theories for Component-based Design, Springer-Verlag (2001) 148-165

40. Negulescu, R.: Process Spaces. In: Proceedings of the 11th Int. Conf. on Concurrency Theory. CONCUR '00, London, UK, UK, Springer-Verlag (2000) 199-213

41. Cofer, D. et al.: Compositional verification of architectural models. In: Proceedings of the 4th International Conference on NASA Formal Methods. NFM'12, Berlin, Heidelberg, Springer-Verlag (2012) 126-140 


\section{A Appendix: Lemmas}

Lemma 1. Given an assertion $\mathrm{W}$ over $X$, it holds that $\operatorname{proj}_{X}(\mathrm{~W})=\mathrm{W}$.

Proof. Consider an assertion W over $X$. In accordance with the relation (1), $\operatorname{proj}_{X}(\mathrm{~W})$ is the set of runs where each run in $\mathrm{W}$ is restricted to the set $X$. Since each run in $\mathrm{W}$ is for $X$, each run in $\operatorname{proj}_{X}(\mathrm{~W})$ is also a run in $\mathrm{W}$, and vice versa. It can hence be concluded that $\operatorname{proj}_{X}(\mathrm{~W})=\mathrm{W}$.

Lemma 2. Given two assertions $\mathrm{W}$ and $\mathrm{W}^{\prime}$, and a set of variables $X^{\prime \prime}$, it holds that $\widehat{\operatorname{proj}}_{X^{\prime \prime}}(\mathrm{W}) \subseteq \widehat{\operatorname{proj}}_{X^{\prime \prime}}\left(\mathrm{W}^{\prime}\right)$, if $\mathrm{W} \subseteq \mathrm{W}^{\prime}$.

Proof. Consider that $\mathrm{W}$ and $\mathrm{W}^{\prime}$ are two assertions over $X$ and $X^{\prime}$, respectively, and $X^{\prime \prime}$ is a set of variables. Given that $\mathrm{W} \widehat{\subseteq} \mathrm{W}^{\prime}$, which is a short-hand notation for $\widehat{\operatorname{proj}}_{X \cup X^{\prime}}(\mathrm{W}) \subseteq \operatorname{proj}_{X \cup X^{\prime}}^{-1}\left(\mathrm{~W}^{\prime}\right)$, in accordance with Sec. 2.1. In accordance with the relation $(2), \widehat{\operatorname{proj}}_{X^{\prime \prime}}\left(\widehat{\operatorname{proj}}_{X \cup X^{\prime}}(\mathrm{W})\right)$ means that each run in $\widehat{p r o j}_{X \cup X^{\prime}}(\mathrm{W})$ is first extended with all possible runs for $X^{\prime \prime} \backslash\left(X \cup X^{\prime}\right)$, and

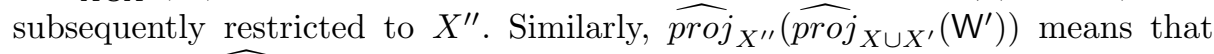
each run in $\widehat{\operatorname{proj}}_{X \cup X^{\prime}}\left(\mathrm{W}^{\prime}\right)$ is first extended with all possible runs for $X^{\prime \prime} \backslash$ $\left(X \cup X^{\prime}\right)$, and subsequently restricted to $X^{\prime \prime}$. Since each run that exists in $\widehat{\operatorname{proj}}_{X \cup X^{\prime}}(\mathrm{W})$, also exists in $\widehat{\operatorname{proj}}_{X \cup X^{\prime}}\left(\mathrm{W}^{\prime}\right)$, and since the runs are extended and restricted in the same manner, each run in $\left.\widehat{\operatorname{proj}_{X^{\prime \prime}}\left(\widehat{\operatorname{proj}}{ }_{X} \cup X^{\prime}\right.}(\mathrm{W})\right)$ will also exist in $\widehat{\operatorname{proj}}{ }_{X^{\prime \prime}}\left(\widehat{\operatorname{proj}}{ }_{X \cup X^{\prime}}\left(\mathrm{W}^{\prime}\right)\right)$. Using the short-hand notation described in Sec. 2.1, it hence holds that $\widehat{\operatorname{proj}}_{X^{\prime \prime}}(\mathrm{W}) \subseteq \widehat{\operatorname{proj}}_{X^{\prime \prime}}\left(\mathrm{W}^{\prime}\right)$.

Lemma 3. An assertion $\mathrm{W}$ over $X$ can only constrain variables that are in $X$.

Proof. Consider an assertion W over $X$. Let $\{x\}$ be a set containing a variable $x$ that is not in $X$. Assume that W constrains $\{x\}$, which will be shown to be contradictory. In accordance with the relation (3), this means that $\mathrm{W} \widehat{\subset} \operatorname{proj}_{X \backslash\{x\}}(\mathrm{W})$. Since $X \backslash\{x\}=X$, Lemma 1 implies that $\operatorname{proj}_{X \backslash\{x\}}(\mathrm{W})=\mathrm{W}$, and this further means that $\mathrm{W} \widehat{\subset} \operatorname{proj}_{X \backslash x}(\mathrm{~W})$ does not hold. It can therefore be concluded that W can only constrain variables that are in $X$.

Lemma 4. Given four assertions $\mathrm{W}, \mathrm{W}^{\prime}, \mathrm{W}^{\prime \prime}$, and $\mathrm{W}^{\prime \prime \prime}$ where $\mathrm{W} \widehat{\cap} \mathrm{W}^{\prime \prime} \widehat{\subseteq} \mathrm{W}^{\prime \prime \prime}$ and $\mathrm{W}^{\prime} \widehat{\subseteq} \mathrm{W}$, it holds that

$$
W^{\prime} \widehat{\cap} W^{\prime \prime} \widehat{\subseteq} W^{\prime \prime \prime} .
$$

Proof. Consider four assertions $\mathrm{W}, \mathrm{W}^{\prime}, \mathrm{W}^{\prime \prime}$, and $\mathrm{W}^{\prime \prime \prime}$ over $X, X^{\prime}, X^{\prime \prime}$, and $X^{\prime \prime \prime}$ where it holds that

$$
\begin{aligned}
& W \widehat{\cap} W^{\prime \prime} \widehat{\subseteq} W^{\prime \prime \prime} \\
& W^{\prime} \widehat{\subseteq} W .
\end{aligned}
$$

In accordance with Sec. 2.1, the relations (64) and (65) can also be written as 


$$
\begin{aligned}
& \widehat{\operatorname{proj}}_{X_{t}}(\mathrm{~W}) \cap \widehat{\operatorname{proj}}_{X_{t}}\left(\mathrm{~W}^{\prime \prime}\right) \subseteq \widehat{\operatorname{proj}}_{X_{t}}\left(\mathrm{~W}^{\prime \prime \prime}\right) \\
& \widehat{\operatorname{proj}}_{X^{\prime} \cup X}\left(\mathrm{~W}^{\prime}\right) \subseteq \widehat{\operatorname{proj}}_{X^{\prime} \cup X}(\mathrm{~W}) \text {, }
\end{aligned}
$$

where $X_{t}=X \cup X^{\prime \prime} \cup X^{\prime \prime \prime}$.

Consider that each run in $\widehat{\operatorname{proj}}_{X_{t}}(\mathrm{~W}), \widehat{\operatorname{proj}}_{X_{t}}\left(\mathrm{~W}^{\prime \prime}\right)$, and $\widehat{\operatorname{proj}} X_{X_{t}}\left(\mathrm{~W}^{\prime \prime}\right)$ are extended with all possible runs for $X^{\prime} \backslash X_{t}$. Considering the relation (66), this would mean that all runs that would be added to $\widehat{\operatorname{proj}_{X}}(\mathrm{~W}) \cap \widehat{\operatorname{proj}}_{X_{t}}\left(\mathrm{~W}^{\prime \prime}\right)$ would also be added to $\widehat{\operatorname{proj}} X_{t}\left(\mathrm{~W}^{\prime \prime \prime}\right)$. This means that it must hold that

$$
\widehat{\operatorname{proj}} X_{X^{\prime} \cup X_{t}}(\mathrm{~W}) \cap \widehat{\operatorname{proj}} \bar{X}_{X^{\prime} \cup X_{t}}\left(\mathrm{~W}^{\prime \prime}\right) \subseteq \widehat{\operatorname{proj}} \widehat{X}_{X^{\prime} \cup X_{t}}\left(\mathrm{~W}^{\prime \prime \prime}\right) .
$$

Similarily, if each run in $\left.\widehat{\operatorname{proj}_{X^{\prime}} \cup X} \mathrm{~W}^{\prime}\right)$ and $\widehat{\operatorname{proj}}_{X^{\prime} \cup X}(\mathrm{~W})$ in the relation (67) are extended with all possible runs for $X_{t} \backslash\left(X^{\prime} \cup X\right)$, it must hold that

$$
\widehat{\operatorname{proj}}_{X^{\prime} \cup X_{t}}\left(\mathrm{~W}^{\prime}\right) \subseteq \widehat{\operatorname{proj}}_{X^{\prime} \cup X_{t}}(\mathrm{~W}) \text {. }
$$

The relations (68) and (69) imply that

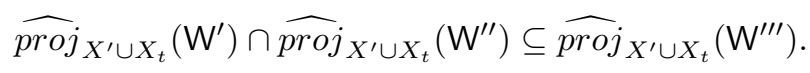

According to Lemma 3 , the assertions $\mathrm{W}^{\prime}, \mathrm{W}^{\prime \prime}$, and $\mathrm{W}^{\prime \prime \prime}$ can only constrain $X^{\prime}, X^{\prime \prime}$, and $X^{\prime \prime \prime}$, respectively. This and the relation (70) imply that $\widehat{\operatorname{proj}}_{X^{\prime} \cup X_{t}}\left(\operatorname{proj}_{X^{\prime}}\left(\mathrm{W}^{\prime}\right)\right) \cap \widehat{\operatorname{proj}}_{X^{\prime} \cup X_{t}}\left(\operatorname{proj}_{X^{\prime \prime}}\left(\mathrm{W}^{\prime \prime}\right)\right) \subseteq \widehat{\operatorname{proj}}_{X^{\prime} \cup X_{t}}\left(\operatorname{proj}_{X^{\prime \prime \prime}}\left(\mathrm{W}^{\prime \prime \prime}\right)\right)$.

In accordance with Sec. 2.1 and Lemma 1, this can also be written as $W^{\prime} \widehat{\cap} W^{\prime \prime} \widehat{\subseteq} W$.

Lemma 5. Given an assertion $\mathrm{W}$, a set of variables $X^{\prime \prime}$, and another set of variables $X^{\prime} \subseteq X^{\prime \prime}$, it holds that $\widehat{\operatorname{proj}}_{X^{\prime \prime}}(\mathrm{W}) \widehat{\subseteq} \widehat{\operatorname{proj}}_{X^{\prime}}(\mathrm{W})$.

Proof. In accordance with the relation (2), given an assertion W over a set of variables $X, \widehat{\operatorname{proj}}_{X^{\prime \prime}}(\mathrm{W})$ is the set of runs where each run in $\mathrm{W}$ is first extended with all possible runs for $X^{\prime \prime} \backslash X$ and subsequently restricted to $X^{\prime \prime}$. Given another set of variables $X^{\prime} \subseteq X^{\prime \prime}, \widehat{\operatorname{proj}}_{X^{\prime}}(\mathrm{W})$ is the set of runs where each run in $\widehat{\operatorname{proj}}_{X^{\prime \prime}}(\mathrm{W})$ is further restricted to $X^{\prime}$. This means that $\widehat{\operatorname{proj}}_{X^{\prime \prime}}\left(\widehat{\operatorname{proj}}_{X^{\prime}}(\mathrm{W})\right)$ is the set of runs where each run in $\widehat{\operatorname{pro}}_{X^{\prime}}(\mathrm{W})$ is extended with all possible runs for $X^{\prime \prime} \backslash X^{\prime}$, while $\widehat{\operatorname{proj}}_{X^{\prime \prime}}(\mathrm{W})$ is the set of runs where each run in $\widehat{\operatorname{proj}}_{X^{\prime}}(\mathrm{W})$ is extended with a subset of all the possible runs for $X^{\prime \prime} \backslash X^{\prime}$. This implies that $\widehat{\operatorname{proj}}_{X^{\prime \prime}}(\mathrm{W}) \subseteq \widehat{\operatorname{proj}}_{X^{\prime \prime}}\left(\widehat{\operatorname{proj}}_{X^{\prime}}(\mathrm{W})\right)$, which can also be written as $\widehat{\operatorname{proj}}_{X^{\prime \prime}}(\mathrm{W}) \widehat{\subseteq} \widehat{\operatorname{proj}}_{X^{\prime}}(\mathrm{W})$ in accordance with Sec. 2.1 .

Lemma 6. Given an assertion $\mathrm{W}$ and a non-empty set of variables $X^{\prime}$, it holds that $\widehat{\operatorname{proj}}_{X^{\prime}}(\mathrm{W}) \neq \emptyset$, if and only if $\mathrm{W} \neq \emptyset$. 
Proof. For the if part, consider a non-empty set of variables $X^{\prime}$ and assume that $\mathrm{W}$ is a non-empty assertion over a set of variables $X$. In accordance with the relation (2), $\widehat{\operatorname{proj}} X_{X^{\prime}}(\mathrm{W})$ is equivalent to $\operatorname{proj}_{X^{\prime}}\left(\widehat{\operatorname{proj}} \bar{X}_{X^{\prime} \cup X}(\mathrm{~W})\right)$. Since $\widehat{\operatorname{proj}} \mathrm{X}_{X^{\prime} \cup X}(\mathrm{~W})$ is obtained by extending each run in $\mathrm{W}$ with all possible runs for $X^{\prime} \backslash X$, it follows from the fact that $W \neq \emptyset$, that

$$
\widehat{\operatorname{proj}}_{X^{\prime} \cup X}(\mathrm{~W}) \neq \emptyset \text {. }
$$

Since $\widehat{\operatorname{proj}} j_{X^{\prime} \cup X}(\mathrm{~W})$ is an assertion over $X^{\prime} \cup X$, Lemma 1 implies that

$$
\widehat{\operatorname{proj}} \bar{X}_{X^{\prime} \cup X}(\mathrm{~W})=\operatorname{proj}_{X^{\prime} \cup X}\left(\widehat{\operatorname{proj}} \bar{X}_{X^{\prime} \cup X}(\mathrm{~W})\right) .
$$

Since $X^{\prime} \subseteq\left(X^{\prime} \cup X\right)$, Lemma 5 implies that

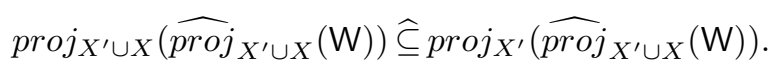

This and the relations (71) and (72) imply that

$$
\operatorname{proj}_{X^{\prime}}\left(\widehat{\operatorname{proj}} j_{X^{\prime} \cup X}(\mathrm{~W})\right) \neq \emptyset \text {. }
$$

Using the short-hand notation as described in Sec. 2.1, it hence holds that $\widehat{\operatorname{proj}}_{X^{\prime}}(\mathrm{W}) \neq \emptyset$.

For the only-if part, consider an assertion $\widehat{\operatorname{proj}_{X^{\prime}}}(\mathrm{W}) \neq \emptyset$. Assume that $\mathrm{W}=$ $\emptyset$, which will be shown to be contradictory. In accordance with the relation (2), $\widehat{\operatorname{proj}_{X^{\prime}}}(\mathrm{W}) \neq \emptyset$ is equivalent to

$$
\operatorname{proj}_{X^{\prime}}\left(\widehat{\operatorname{proj}} \bar{X}^{\prime} \cup X(\mathrm{~W})\right) \neq \emptyset \text {. }
$$

Since $W$ has no runs and $\widehat{\operatorname{proj}} j_{X^{\prime} \cup X}(\mathrm{~W})$ is the set of runs where each runs in W is extended with all possible runs for $X^{\prime} \backslash X$, it follows that $\widehat{p r o} j_{X^{\prime} \cup X}(\mathrm{~W})=\emptyset$.

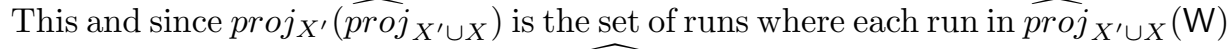

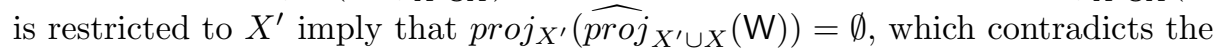
relation (73). It hence follows that it must hold that $W \neq \emptyset$, which concludes the only-if part of the proof.

Lemma 7. Given three assertions $\mathrm{W}, \mathrm{W}^{\prime}$, and $\mathrm{W}^{\prime \prime}$ where $\mathrm{W} \widehat{\cap} \mathrm{W}^{\prime \prime} \neq \emptyset$ and $\mathrm{W} \widehat{\subseteq} \mathrm{W}^{\prime}$, it holds that

$$
W^{\prime} \widehat{\cap} W^{\prime \prime} \neq \emptyset \text {. }
$$

Proof. Consider four assertions $\mathrm{W}, \mathrm{W}^{\prime}, \mathrm{W}^{\prime \prime}$, and $\mathrm{W}^{\prime \prime \prime}$ over $X, X^{\prime}, X^{\prime \prime}$, and $X^{\prime \prime \prime}$, respectively, where it holds that $W \widehat{\cap} W^{\prime \prime} \neq \emptyset$ and $W \widehat{\subseteq} W^{\prime}$. In accordance with Sec. $2.1, W \widehat{\cap} W^{\prime \prime} \neq \emptyset$ can also be written as

$$
\widehat{\operatorname{proj}}{ }_{X \cup X^{\prime \prime}}(\mathrm{W}) \cap \widehat{\operatorname{proj}}{ }_{X \cup X^{\prime \prime}}\left(\mathrm{W}^{\prime \prime}\right) \neq \emptyset \text {. }
$$

Consider that each run in the assertions $\widehat{\operatorname{proj}}{ }_{X \cup X^{\prime \prime}}(\mathrm{W})$ and $\widehat{\operatorname{proj}_{X} \cup X^{\prime \prime}}\left(\mathrm{W}^{\prime \prime}\right)$ in the relation (74) are extended with all possible run for $X^{\prime} \backslash\left(X \cup X^{\prime \prime}\right)$. Since 
each run that is added to $\widehat{\operatorname{proj}_{X} \cup X^{\prime \prime}}(\mathrm{W})$ is also added to $\widehat{\operatorname{proj}_{X} \cup X^{\prime \prime}}\left(\mathrm{W}^{\prime \prime}\right)$, it holds that

$$
\widehat{\operatorname{proj}}_{X \cup X^{\prime \prime} \cup X^{\prime}}(\mathrm{W}) \cap \widehat{\operatorname{proj}}_{X \cup X^{\prime \prime} \cup X^{\prime}}\left(\mathrm{W}^{\prime \prime}\right) \neq \emptyset .
$$

Lemma 2 and since it was given that $\mathrm{W} \widehat{\subseteq} \mathrm{W}^{\prime}$ imply that

$$
\widehat{\operatorname{proj}}_{X \cup X^{\prime \prime} \cup X^{\prime}}(\mathrm{W}) \subseteq \widehat{\operatorname{proj}}_{X \cup X^{\prime \prime} \cup X^{\prime}}\left(\mathrm{W}^{\prime}\right) .
$$

This and the relation (75) imply that $\widehat{\operatorname{proj}} j_{X \cup X^{\prime \prime} \cup X^{\prime}}\left(\mathrm{W}^{\prime}\right) \cap \widehat{\operatorname{proj}}{ }_{X \cup X^{\prime \prime} \cup X^{\prime}}\left(\mathrm{W}^{\prime \prime}\right) \neq$ $\emptyset$. In accordance with Sec. 2.1, this can also be written as $W^{\prime} \hat{\cap} W^{\prime \prime} \neq \emptyset$.

Lemma 8. Given three assertions $\mathrm{W}, \mathrm{W}^{\prime}$, and $\mathrm{W}^{\prime \prime}$ where $\emptyset \subset \mathrm{W} \widehat{\cap} \mathrm{W}^{\prime} \subseteq \mathrm{W}^{\prime \prime}$, it holds that

$$
\mathrm{W} \widehat{\cap} \mathrm{W}^{\prime \prime} \neq \emptyset \text {. }
$$

Proof. Consider three assertions $\mathrm{W}, \mathrm{W}^{\prime}$, and $\mathrm{W}^{\prime \prime}$ over $X, X^{\prime}$, and $X^{\prime \prime}$, respectively, where it holds that

$$
\emptyset \subset \mathrm{W} \widehat{\cap} \mathrm{W}^{\prime} \widehat{\subseteq} \mathrm{W}^{\prime \prime}
$$

This and since $\left(W \widehat{\cap} W^{\prime}\right) \hat{\cap}\left(W \widehat{\cap} W^{\prime}\right)=W \widehat{\cap} W^{\prime}$ imply that it holds that $\emptyset \subset$ $\left(W \widehat{\cap} W^{\prime}\right) \widehat{\cap}\left(W \widehat{\cap} W^{\prime}\right) \widehat{\subseteq} W^{\prime \prime}$. This, the relation (76), and Lemma 7 imply that $\left(W \hat{\cap} W^{\prime}\right) \hat{\cap} W^{\prime \prime} \neq \emptyset$. This implies that $W^{\prime \prime} \neq \emptyset$.

Lemma 9. Given two assertions $\mathrm{W}$ and $\mathrm{W}^{\prime}$ where $\mathrm{W} \widehat{\subseteq} \mathrm{W}^{\prime}$, it holds that the set of variables constrained by $\mathrm{W}^{\prime}$ is a subset of the set of variables constrained by W.

Proof. Consider two assertions $\mathrm{W}$ and $\mathrm{W}^{\prime}$ over $X$ and $X^{\prime}$, respectively where

$$
\mathrm{W} \widehat{\subseteq} \mathrm{W}^{\prime} .
$$

In accordance with Sec. 2.1, this can also be written as

$$
\widehat{\operatorname{proj}}_{X \cup X^{\prime}}(\mathrm{W}) \widehat{\subseteq} \widehat{\operatorname{proj}}_{X \cup X^{\prime}}\left(\mathrm{W}^{\prime}\right) \text {. }
$$

Assume that $W^{\prime}$ constrains $\left\{x^{\prime}\right\}$. In accordance with the relation (3), this means that

$$
\begin{cases}\mathrm{W}^{\prime} \widehat{\subset} \operatorname{proj}_{X^{\prime} \backslash\left\{x^{\prime}\right\}}\left(\mathrm{W}^{\prime}\right) & X^{\prime} \backslash\left\{x^{\prime}\right\} \neq \emptyset \\ \mathrm{W}^{\prime} \subset \Omega_{\left\{x^{\prime}\right\}} & \text { otherwise }\end{cases}
$$

This, Lemma 4 and the relation (77) imply that

$$
\begin{cases}\mathrm{W} \widehat{\subset} \operatorname{proj}_{X^{\prime} \backslash\left\{x^{\prime}\right\}}\left(\mathrm{W}^{\prime}\right) & X^{\prime} \backslash\left\{x^{\prime}\right\} \neq \emptyset \\ \mathrm{W} \subset \Omega_{\left\{x^{\prime}\right\}} & \text { otherwise }\end{cases}
$$

Assume the contradictory relation

$$
\begin{cases}\mathrm{W} \not \subset \operatorname{proj}_{X \backslash\left\{x^{\prime}\right\}}(\mathrm{W}) & X \backslash\left\{x^{\prime}\right\} \neq \emptyset \\ \mathrm{W} \not \subset \Omega_{\left\{x^{\prime}\right\}} & \text { otherwise }\end{cases}
$$


The relation (77) and Lemma 2 imply that

$$
\operatorname{proj}_{X \backslash\left\{x^{\prime}\right\}}(\mathrm{W}) \subseteq \operatorname{proj}_{X \backslash\left\{x^{\prime}\right\}}\left(\mathrm{W}^{\prime}\right) .
$$

This and the relation (80) imply that

$$
\begin{cases}\mathrm{W} \not \subset \operatorname{proj}_{X^{\prime} \backslash\left\{x^{\prime}\right\}}\left(\mathrm{W}^{\prime}\right) & X^{\prime} \backslash\left\{x^{\prime}\right\} \neq \emptyset \\ \mathrm{W} \not \subset \Omega_{\left\{x^{\prime}\right\}} & \text { otherwise }\end{cases}
$$

which contradicts the relation (79). This hence means that it must hold that

$$
\begin{cases}\mathrm{W} \widehat{\subset} \operatorname{proj}_{X \backslash\left\{x^{\prime}\right\}}(\mathrm{W}) & X \backslash\left\{x^{\prime}\right\} \neq \emptyset \\ \mathrm{W} \subset \Omega_{\left\{x^{\prime}\right\}} & \text { otherwise. }\end{cases}
$$

In accordance with the relation (3), this means that $W$ constrains $\left\{x^{\prime}\right\}$.

Lemma 10. Given three assertions $\mathrm{W}, \mathrm{W}^{\prime}$, and $\mathrm{W}^{\prime \prime}$ over $X, X^{\prime}$, and $X^{\prime \prime}$, respectively, and a set of variables $Y$ such that $Y \cap\left(X^{\prime} \cup X^{\prime \prime}\right)=\emptyset$, it holds that $\mathrm{W}^{\prime} \widehat{\cap} \operatorname{proj}_{X \backslash Y}(\mathrm{~W}) \widehat{\subseteq} \mathrm{W}^{\prime \prime}$, if and only if $\mathrm{W}^{\prime} \widehat{\cap} \mathrm{W} \widehat{\subseteq} \mathrm{W}^{\prime \prime}$.

Proof. For the if part, assume that $\mathrm{W} \widehat{\cap} \mathrm{W}^{\prime} \widehat{\subseteq} \mathrm{W}^{\prime \prime}$ where $\mathrm{W}, \mathrm{W}^{\prime}$, and $\mathrm{W}^{\prime \prime}$ are assertions over $X, X^{\prime}$, and $X^{\prime \prime}$, respectively. Lemma 2 and Lemma 1 imply that

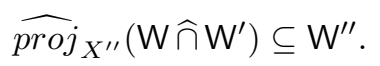

As described in Sec. 2.1, W $\widehat{\cap} \mathrm{W}^{\prime}$ is the set of runs where each run that is in both $\operatorname{proj}_{X \cap X^{\prime}}(\mathrm{W})$ and $\operatorname{proj}_{X \cap X^{\prime}}(\mathrm{W})$ is extended with at least one run for $X^{\prime} \backslash X$ and at least one run for $X \backslash X^{\prime}$.

In comparison, consider the assertion $\operatorname{proj}_{X \backslash Y}(\mathrm{~W}) \widehat{\cap} \mathrm{W}^{\prime}$ where $Y$ is a set of variables such that $Y \cap\left(X^{\prime} \cup X^{\prime \prime}\right)=\emptyset$. Since $\left(X \cap X^{\prime}\right) \subseteq(X \backslash Y), \operatorname{proj}_{X \cap X^{\prime}}(\mathrm{W})$ is the set run of runs where each run in $\operatorname{proj}_{X \backslash Y}(\mathrm{~W})$ is further restricted to $X \cap X^{\prime}$, i.e. that $\operatorname{proj}_{X \cap X^{\prime}}\left(\operatorname{proj}_{X \backslash Y}(\mathrm{~W})\right)=\operatorname{proj}_{X \cap X^{\prime}}\left(\mathrm{W} \widehat{\cap} \mathrm{W}^{\prime}\right)$. This means that $\operatorname{proj}_{X \backslash Y}(\mathrm{~W}) \widehat{\cap} \mathrm{W}^{\prime}$ is the set of runs where each run that is in both $\operatorname{proj}_{X \cap X^{\prime}}(\mathrm{W})$ and $\operatorname{proj}_{X \cap X^{\prime}}(\mathrm{W})$ is extended with the same runs for $X^{\prime} \backslash X$ as for the case of $\mathrm{W} \widehat{\cap} \mathrm{W}^{\prime}$, but where the runs for $X \backslash X^{\prime}$ are further restricted to $X \backslash\left(X^{\prime} \cup Y\right)$. Formulated differently, $\operatorname{proj}_{X \backslash Y}(\mathrm{~W}) \widehat{\cap} \mathrm{W}^{\prime}$ is the set of runs where each run in $\mathrm{W} \widehat{\cap} \mathrm{W}^{\prime}$ is restricted to $\left(X \cup X^{\prime}\right) \backslash Y$, i.e.

$$
\operatorname{proj}_{X \backslash Y}(\mathrm{~W}) \widehat{\cap} \mathrm{W}^{\prime}=\operatorname{proj}_{\left(X \cup X^{\prime}\right) \backslash Y}\left(\mathrm{~W} \widehat{\cap} \mathrm{W}^{\prime}\right) .
$$

Since Lemma 3 implies that $\mathrm{W} \widehat{\cap} \mathrm{W}^{\prime}$ can only constrain variables in $X \cup X^{\prime}$, it holds that if $\widehat{\operatorname{proj}_{X^{\prime \prime}}}\left(\mathrm{W} \widehat{\cap} \mathrm{W}^{\prime}\right)$ is the set of runs where each run in $\mathrm{W} \widehat{\cap} \mathrm{W}^{\prime}$ is restricted to $X^{\prime \prime} \cap\left(X \cup X^{\prime}\right)$ and then subsequently extended with all runs for $X^{\prime \prime} \backslash\left(X \cup X^{\prime}\right)$, i.e. it holds that

$$
\widehat{\operatorname{proj}} X_{X^{\prime \prime}}\left(\mathrm{W} \widehat{\cap} \mathrm{W}^{\prime}\right)=\widehat{\operatorname{proj}} X_{X^{\prime \prime}}\left(\operatorname{proj}_{X^{\prime \prime} \cap\left(X \cup X^{\prime}\right)}\left(\mathrm{W} \widehat{\cap} \mathrm{W}^{\prime}\right)\right) \text {. }
$$


Since $\left(X^{\prime \prime} \cap\left(X \cup X^{\prime}\right)\right) \subset\left(X \cup X^{\prime}\right) \backslash Y$, it is true that $\operatorname{proj}_{X^{\prime \prime} \cap\left(X \cup X^{\prime}\right)}\left(\mathrm{W} \cap \mathrm{W}^{\prime}\right)$ is the set of runs where each run in $\operatorname{proj}_{\left(X \cup X^{\prime}\right) \backslash Y}\left(\mathrm{~W} \widehat{\cap} \mathrm{W}^{\prime}\right)$ is further restricted to $X^{\prime \prime} \cap\left(X \cup X^{\prime}\right)$. This and the relation 82 imply that

$$
\operatorname{proj}_{X^{\prime \prime} \cap\left(X \cup X^{\prime}\right)}\left(\mathrm{W} \widehat{\cap} \mathrm{W}^{\prime}\right)=\operatorname{proj}_{X^{\prime \prime} \cap\left(X \cup X^{\prime}\right)}\left(\operatorname{proj}_{X \backslash Y}(\mathrm{~W}) \widehat{\cap} \mathrm{W}^{\prime}\right) .
$$

This and the relations (81) and (83) imply that

$$
\widehat{\operatorname{proj}}_{X^{\prime \prime}}\left(\operatorname{proj}_{X^{\prime \prime} \cap\left(X \cup X^{\prime}\right)}\left(\operatorname{proj}_{X \backslash Y}(\mathrm{~W}) \widehat{\cap} \mathrm{W}^{\prime}\right)\right) \subseteq \mathrm{W}^{\prime \prime},
$$

which is, in accordance with Sec. 2.1, equivalent to

$$
\widehat{\operatorname{proj}}_{X^{\prime \prime}}\left(\operatorname{proj}_{X \backslash Y}(\mathrm{~W}) \hat{\cap} \mathrm{W}^{\prime}\right) \subseteq \mathrm{W}^{\prime \prime} .
$$

Since $X^{\prime \prime} \subseteq X^{\prime \prime} \cup(X \backslash Y) \cup X^{\prime}$, Lemma 5 implies that

$$
\begin{gathered}
\operatorname{proj}_{X^{\prime \prime} \cup(X \backslash Y) \cup X^{\prime}}\left(\operatorname{proj}_{X \backslash Y}(\mathrm{~W}) \widehat{\cap} \mathrm{W}^{\prime}\right) \subseteq \\
\widehat{\operatorname{proj}}_{X^{\prime \prime} \cup(X \backslash Y) \cup X^{\prime}}\left(\widehat{\operatorname{proj}_{X^{\prime \prime}}}\left(\operatorname{proj}_{X \backslash Y}(\mathrm{~W}) \hat{\cap} \mathrm{W}^{\prime}\right)\right) .
\end{gathered}
$$

This and the relation (84) implies that $\operatorname{proj}_{X^{\prime \prime} \cup(X \backslash Y) \cup X^{\prime}}\left(\operatorname{proj}_{X \backslash Y}(\mathrm{~W}) \widehat{\cap} \mathrm{W}^{\prime}\right) \subseteq$ $\widehat{\operatorname{proj}}_{X^{\prime \prime} \cup(X \backslash Y) \cup X^{\prime}}\left(\mathrm{W}^{\prime \prime}\right)$. This and since $\operatorname{proj}_{X \backslash Y}(\mathrm{~W}) \widehat{\cap} \mathrm{W}^{\prime}$ is an assertion over $X^{\prime \prime} \cup(X \backslash Y) \cup X^{\prime}$ and Lemma 1 hence implies that

$$
\operatorname{proj}_{X^{\prime \prime} \cup(X \backslash Y) \cup X^{\prime}}\left(\operatorname{proj}_{X \backslash Y}(\mathrm{~W}) \widehat{\cap} \mathrm{W}^{\prime}\right)=\operatorname{proj}_{X \backslash Y}(\mathrm{~W}) \hat{\cap} \mathrm{W}^{\prime},
$$

it holds that $\operatorname{proj}_{X \backslash Y}(\mathrm{~W}) \widehat{\cap} \mathrm{W}^{\prime} \subseteq \widehat{\operatorname{proj}}_{X^{\prime \prime} \cup(X \backslash Y) \cup X^{\prime}}\left(\mathrm{W}^{\prime \prime}\right)$. In accordance with Sec. 2.1, this can also be written as $\operatorname{proj}_{X \backslash Y}(\mathrm{~W}) \widehat{\cap} \mathrm{W}^{\prime} \widehat{\subseteq} \mathrm{W}^{\prime \prime}$, which concludes the if part of the proof.

For the if-only part, consider three assertions $\mathrm{W}, \mathrm{W}^{\prime}$, and $\mathrm{W}^{\prime \prime}$ over $X, X^{\prime}$, and $X^{\prime \prime}$, respectively, and a set of variables $Y$ such that $Y \cap\left(X^{\prime} \cup X^{\prime \prime}\right)=\emptyset$, where $\mathrm{W}^{\prime} \widehat{\cap} \operatorname{proj}_{X \backslash Y}(\mathrm{~W}) \widehat{\subseteq} \mathrm{W}^{\prime \prime}$. Since Lemma 1 implies that $\operatorname{proj}_{X}(\mathrm{~W})=\mathrm{W}$ and Lemma 5 implies that $\operatorname{proj}_{X}(\mathrm{~W}) \widehat{\subseteq} \operatorname{proj}_{X \backslash Y}(\mathrm{~W})$, it holds that $\mathrm{W} \widehat{\cap} \mathrm{W}^{\prime} \widehat{\subseteq} \mathrm{W}^{\prime \prime}$, which completes the if-only part of the proof.

Lemma 11. Given four assertions $\mathrm{W}, \mathrm{W}^{\prime}, \mathrm{W}^{\prime \prime}$, and $\mathrm{W}^{\prime \prime \prime}$ where $\mathrm{W} \widehat{\subseteq} \mathrm{W}^{\prime}$ and $\mathrm{W}^{\prime \prime} \widehat{\subseteq} \mathrm{W}^{\prime \prime \prime}$, it holds that $\mathrm{W} \widehat{\cap} \mathrm{W}^{\prime \prime} \subseteq \mathrm{W}^{\prime} \widehat{\cap} \mathrm{W}^{\prime \prime \prime}$.

Proof. Consider four assertions $\mathrm{W}, \mathrm{W}^{\prime}, \mathrm{W}^{\prime \prime}$, and $\mathrm{W}^{\prime \prime \prime}$ over $X, X^{\prime}, X^{\prime \prime}$, and $X^{\prime \prime \prime}$, respectively, where it holds that $\mathrm{W} \widehat{\subseteq} \mathrm{W}^{\prime}$ and $\mathrm{W}^{\prime \prime} \widehat{\widehat{\subseteq}} \mathrm{W}^{\prime \prime \prime}$. This and Lemma 2 imply that it holds that $\widehat{\operatorname{proj}}_{X^{t}}(\mathrm{~W}) \subseteq \widehat{\operatorname{proj}}_{X^{t}}\left(\mathrm{~W}^{\prime}\right)$ and $\widehat{\operatorname{proj}}_{X^{t}}\left(\mathrm{~W}^{\prime \prime}\right) \subseteq \widehat{\operatorname{proj}}_{X^{t}}\left(\mathrm{~W}^{\prime \prime \prime}\right)$ where $X^{t}=X \cup X^{\prime} \cup X^{\prime \prime} \cup X^{\prime \prime \prime}$. This implies that $\widehat{\operatorname{proj}}_{X^{t}}(\mathrm{~W}) \cap \widehat{\operatorname{proj}}_{X^{t}}\left(\mathrm{~W}^{\prime \prime}\right) \subseteq$ $\widehat{\operatorname{proj}}_{X^{t}}\left(\mathrm{~W}^{\prime}\right) \cap \widehat{\operatorname{proj}}_{X^{t}}\left(\mathrm{~W}^{\prime \prime \prime}\right)$. In accordance with Sec. 2.1, this can also be written as $W^{n} W^{\prime \prime} \hat{\subseteq} W^{\prime} \hat{\cap} W^{\prime \prime \prime}$. 